\title{
El tesoro de la villa romana de Boca do Rio (Vila do Bispo, Algarve, Portugal): 90 años después de su descubrimiento
}

\author{
The hoard of the Roman villa of Boca do Rio \\ (Vila do Bispo, Algarve, Portugal): 90 years \\ after its discovery
}

\author{
NOÉ CONEJO DELGADO \\ Departamento de Prehistoria y Arqueología \\ Universidad de Sevilla \\ María de Padilla $\mathrm{s} / \mathrm{n}$ \\ 41004 Sevilla \\ nconejo@us.es \\ https://orcid.org/0000-0002-4367-5695
}

Resumen

El tesoro de la villa romana de Boca do Rio (Vila do Bispo, Algarve, Portugal) es un conjunto monetario del siglo IV hallado en el interior de un ánfora en los años 30 del siglo XX. En su origen este tesoro contaba con más de 1000 monedas, sin embargo tan solo se conocen hoy 284 piezas, las cuales han sido estudiadas de manera parcial por algunos investigadores. Desde un planteamiento actual, se ha reunido toda la información disponible sobre las circunstancias que ocasionaron el descubrimiento de este tesoro, con la intención de aportar mayor precisión sobre cuándo y porqué fue ocultado. Así mismo, se ha realizado un estudio pormenorizado de las 284 piezas con el objetivo de caracterizar mejor el conjunto; y se ha comparado este con otros tesoros coetáneos y similares documentados en villae lusitanas, con la intención de aportar una nueva reflexión sobre el rol de la moneda en las economías rurales tardo-antiguas de Lusitania.

Palabras clave: Lusitania, villae, moneda bronce, tesoro, economía rural

\section{Abstract}

The hoard of Roman villa of Boca do Rio (Vila do Bispo, Algarve, Portugal) is a monetary group from the $4^{\text {th }}$ century found inside an amphora in the 30's of the $20^{\text {th }}$ century. In the beginning this hoard had more than 1,000 coins, however only 284 pieces are known today, which have been partially studied by some researchers. From a current perspective, I have compiled all the information on the circumstances of the discovery of this hoard, with the intention of providing more precise information on why it was concealment. I have also proposed a detailed study of the 284 pieces in order to better characterize this hoard; and I have compared the Boca do Rio hoard with other contemporary and similar hoards documented in lusitanian villas, with the intention of contributing a new reflection on the role of the currency in the Late-antiquity rural economies of Lusitania.

Key words: Lusitania, villae, bronze currency, hoard, rural economy 


\section{Introducción}

En 1933 aparecieron más de 1000 monedas de bronce, acuñadas entre los siglos III y IV d. C., en el proceso de extracción de los pavimentos musivos de una de las estancias de la villa romana de Boca do Rio. Desde ese momento hasta hoy, muy pocos investigadores se han aproximado a este conjunto monetario del que solo se conservan 284 piezas. El estudio de estos ejemplares y su comparación con otros conjuntos similares nos permitirá plantear una contextualización histórica de tal depósito, del cual carecemos de documentación estratigráfica dado el carácter fortuito de su descubrimiento.

El conjunto de piezas de Boca do Rio se suma al considerable número de casos similares documentados en las villae de Lusitania. Un amplio grupo de hallazgos que no han recibido numerosas atenciones en los últimos años; y mucho menos aún, a las preguntas que surgen en torno a ellos: por qué fueron depositados y por qué nunca fueron recuperados. El estudio del conjunto de Boca do Rio nos ayudará a reflexionar sobre esas dos cuestiones y lanzar nuevas hipótesis.

\section{Boca do Rio: una villa en el litoral lusitano}

La villa romana de Boca do Rio está ubicada a $5 \mathrm{~km}$ al sureste de la actual población de Budens, freguesia del concejo de Vila do Bispo, distrito de Faro, Portugal (figura I). Las ruinas habían sido descritas por varios autores ya en el siglo XviII (Bernardes y Medeiros, 2016: 266) y su estructura es conocida gracias a los trabajos arqueológicos efectuados por Estacio da Veiga a finales del siglo xIx, quien publicó en rgio una descripción detallada de su excavación y un dibujo de la planta exhumada (da Veiga, I9ıо: 2I2-216, lám. 3; Fabião, 2019: 96) (figura 2). En estos trabajos, E. da Veiga — quien creía encontrarse ante una ciudad desconocida de época romana y no ante una villa - identificó un gran número de estancias de forma cuadrangular y rectangular con pavimentos de mosaicos (estancias A-C, E-G). Estas formaban parte de un mismo edificio y estaban dispuestas a lo largo de un pasillo rectangular con función de corredor (estancia D) que conectaba las estancias anteriores con otras de forma similar; alguna de ellas, con cabecera en ábside (estancias I-N). Más alejadas, pero seguramente conectadas con las anteriores por el mismo corredor, existían otras dos estructuras destinadas — según E. da Veiga - a la producción de conservas de pescado (estancias $\mathrm{Q}$ y P) (da Veiga, I9ıо: 212-216, lám. 3).

Sobre las estructuras identificadas por E. da Veiga, otros arqueólogos continuaron trabajos de excavación y extracción de mosaicos a finales del siglo xIx y durante la primera mitad del siglo xx. Estas tareas proporcionaron la mayor parte de los materiales arqueológicos conocidos hoy de este yacimiento, los cuales se hallan conservados en varios museos portugueses (Viana et alii, 1953: I19-124; Santos, 1971: 84-95; Bernardes y Medeiros, 2016: 266-267). Pero además, estas labores de investigación también demostraron que las estructuras ubicadas en la playa de Boca do Rio eran parte del área residencial de una villa, posiblemente de corredor, con un amplio aparato decorativo compuesto por columnas de mármol, mosaicos de gran calidad y estucos con pinturas polícromas (Santos, 1971: 84).

Estas estancias han sido objeto de varias lecturas a lo largo de los años, existiendo múltiples interpretaciones sobre su utilidad; fundamentalmente sobre las que más elementos decorativos han proporcionado (K-O). De este modo, algunos investigadores que han partido de los dibujos y descripciones de E. da Veiga pudieron situar en esa área un modesto complejo termal del que solo podían identificarse, por la pérdida de estructuras, las salas de agua fría (Reis, 2004: I22-I23; García-Entero, 2006: 44I-442). Recientemente J.P. Bernardes e I. Medeiros (2016: 284-285) han propuesto una nueva interpretación de los planos proporcionados por E. da Veiga en I9ı。; a la luz de recientes excavaciones que ellos mismos han dirigido y con el hándicap de que gran parte del yacimiento ya había sido destruido por la erosión marina (figura 3). Así pues, la proposición de los autores interpreta el área comprendida por las estancias $\mathrm{K}-\mathrm{O}$ como la pars urbana de la villa, la cual estaría conectada por el corredor (estancia D) al espacio ocupado por las termas (estancias A y B) y a otras salas dedicadas 


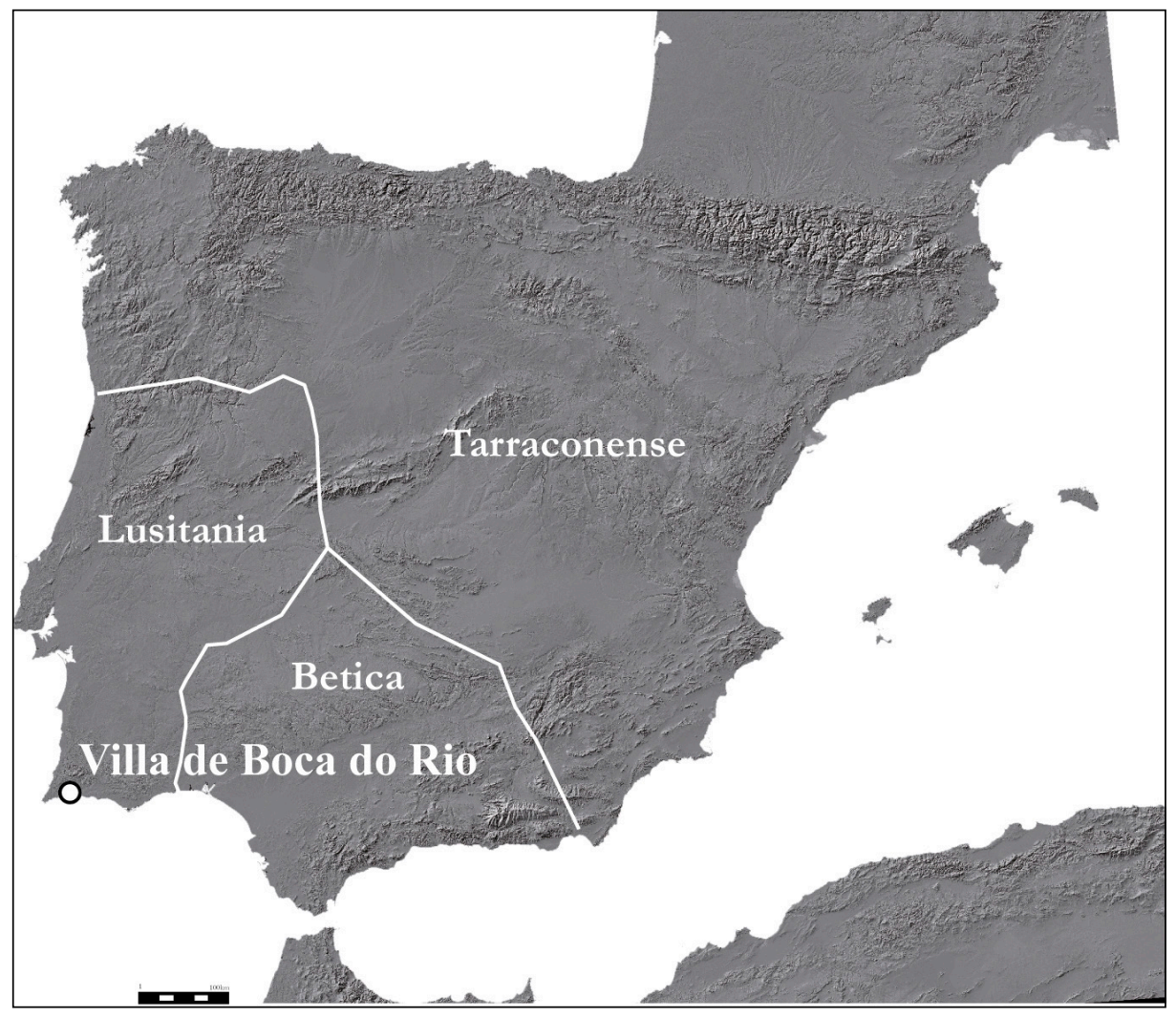

Figura 1. Localización de la villa romana de Boca do Rio

Figure 1. Location of the Roman villae of Boca do Rio

a actividades domésticas como cocina y almacenes (estancias C, E-H).

Por lo que respecta a las estructuras $\mathrm{P}$ y Q identificadas por E. da Veiga, estas consistían en amplios tanques construidos en buena fábrica y destinados a la producción de conservas de pescado (da Veiga, I9IO: 2I2). Este hecho fue verificado posteriormente con otras intervenciones arqueológicas realizadas en los años treinta por J. Formosinho, y más recientemente por otros arqueólogos (Alves, 1997; Bernardes, 2007; Bernardes y Medeiros, 20I7), quienes identificaron además otras estructuras productivas situadas a varios metros de distancia del edificio. La asociación de estas a la villa demostró que uno de los principales sustentos económicos de este yacimiento litoral era la producción de conservas de pescado, como también sucedía en otros sitios coetáneos y próximos (Lagóstena, 200I: 230; Medeiros, 20I4-20I5).

A pesar de las detalladas descripciones ofrecidas por E. da Veiga, sus trabajos no permitieron a arqueólogos posteriores poder identificar las diferentes fases de ocupación desarrolladas en Boca do Rio.
El estudio de materiales realizado por J.P. Bernardes e I. Medeiros (Bernardes y Medeiros, 20I6: 280-28I) han posibilitado una datación ajustada, al menos de las últimas fases de ocupación. De este modo, ambos arqueólogos sostienen que la villa fue fundada durante el Alto Imperio, posiblemente en la primera mitad del siglo i d. C. por el hallazgo de cerámicas fácilmente datadas en ese horizonte (TSSudg, TSI y TSHisp). Posteriormente, ya en la segunda mitad del siglo in y durante el siglo iv, Boca do Rio experimenta un gran apogeo como así demuestra un amplio consumo de cerámicas procedentes de talleres africanos. Es muy probable que durante esta fase se produjera la ampliación del edificio, la decoración de sus paredes con estucos y la dotación de pavimentos musivos a las principales salas. Un comportamiento muy similar al observado en el resto de Hispania (Chavarría, 2007: 94-95; Gorges 2008: 39-40) y en Lusitania, con ejemplos evidentes en el Alentejo con Quinda das Longas (Carvalho y Almeida, 2005: 379-383), Torre de Palma (Maloney y Hale, I996: 285-286) y Horta da Torre 


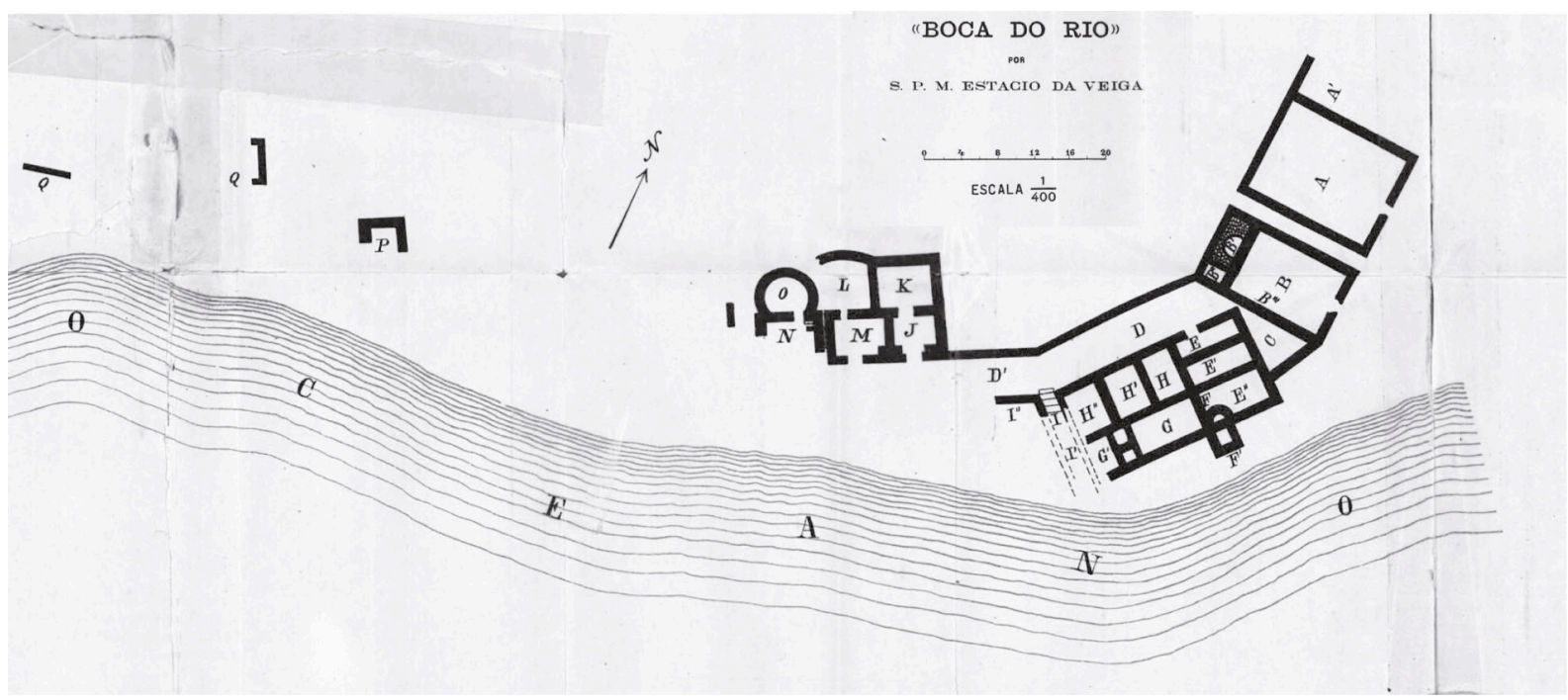

Figura 2. Planta exhumada en las excavaciones de Estacio da Veiga a finales del siglo XIX. (da Veiga, 1910: lám. 3)

Figure 2. Plany of the Estacio da Veiga excavations at the end of the $19^{\text {th }}$ century

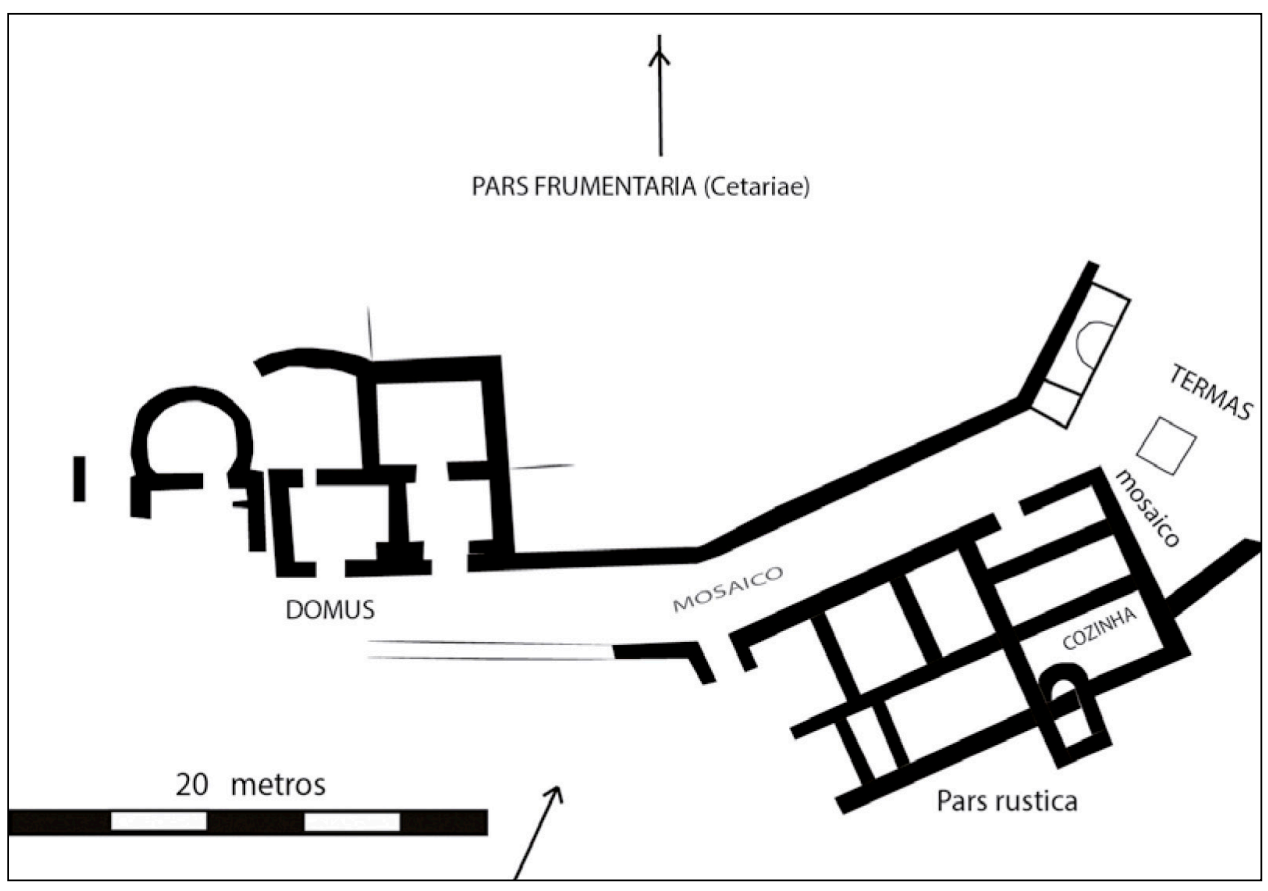

Figura 3. Nueva interpretación de las estructuras (Bernardes y Medeiros, 2016: 284, fig. 19)

Figure 3. New interpretation of the Estacio da Veiga Plant (Bernardes and Medeiros, 2016: 284, fig. 19)

(Carneiro, 20I7); y ya en el Algarve, con los casos de Milreu y Cerro da Vila (Teichner, 2006).

Durante las primeras décadas del siglo $\mathrm{v}-\mathrm{mo}^{-}$ mento en el que pudo ser ocultado el tesoro que describiremos a continuación - la villa muestra signos evidentes de abandono en la pars urbana. Esta parte fue reaprovechada por otras personas que llevaron a cabo el encalado de los estucos de las salas más nobles, y realizaron algunas reparaciones toscas en los pavimentos de mosaico (Bernardes y Medeiros, 20r6: 283). Este comportamiento puede ser similar al observado en otros yacimientos cercanos y de la misma época, en los cuales una vez la pars urbana ha perdido la función residencial, varios grupos de personas reocupan tales áreas, haciendo de las estancias ricamente decoradas y de los peristilos, nuevos espacios domésticos. Esto no solo ha sido observado en las villae lusitanas de Freiria 
(Cardoso, 2016: 512), Frielas (Quaresma, 2017a: 433), Quinta das Longas (Carneiro, 20r6: 297) o en las vecinas Milreu (Teichner, 2006: 2I2) y Cerro da Vila (Teichner, 20I7: 424); sino también en la mayoría de los casos hispanos (Chavarría, 2013: 140).

La ausencia de fragmentos cerámicos de TSFT en el registro de materiales hace situar el abandono de la villa en la primera mitad del siglo $\mathrm{v}$ (Bernardes y Medeiros, 20I6: 280). Este tipo cerámico es muy frecuente en otros yacimientos algarvíos, y permite a los arqueólogos datar las ocupaciones tardías incluso hasta el siglo vi (Fabião, 2009: 34), como así se ha observado en Milreu y Cerro da Vila (Teichner, 2006: 2I2). Autores como I. Medeiros han expuesto que el abandono de Boca do Rio puede responder a diversos factores entre los que figurarían el impacto de las invasiones bárbaras - factor que incluso el mismo autor descarta por su escasa incidencia en el sur de Lusitania-, inclemencias marítimas o la colmatación del estuario donde se ubica el yacimiento, haciéndose inviable las actividades económicas (Medeiros, 2014-2015: 140). Un cese de la actividad que sin duda no responde a consecuencias de acciones violentas, y que encaja perfectamente con un abandono consensuado por la falta de recursos aprovechables que anteriormente ofertaba el entorno.

\section{El Tesoro de Boca do Rio}

\subsection{Algunos datos sobre su historia}

El carácter fortuito del hallazgo de este conjunto monetario no nos ha proporcionado información estratigráfica; muy interesante sin duda para conocer entre otras cosas las circunstancias en las que este tesoro fue ocultado. No obstante, creemos que disponemos de ciertos datos que nos pueden ayudar a conocer las circunstancias del descubrimiento y a completar la información inconexa que existe sobre él.

La figura del doctor J. Formosinho está completamente ligada a la historia de Boca do Rio y del citado tesoro. Este notario público oriundo de Lagos fue el impulsor de la creación de un museo regional en su localidad natal, cuyo objetivo principal era exponer las piezas arqueológicas más significativas halladas en el sur de Portugal. La apertura de esta institución, de la cual el doctor Formosinho fue conservador y director hasta 1960, implicó el estudio y excavación o reexcavación de un gran número de yacimientos situados en la parte más occidental del Algarve, con la intención de enriquecer los fondos del recién abierto museo. En este sentido, y con el deseo de exhumar parte de los mosaicos documentados por E. da Veiga en Boca do Rio, el doctor Formosinho lleva a cabo varias intervenciones sobre este yacimiento en 1933 (Formosinho, I997: 60). Parece que durante la reexcavación de la sala K del plano de E. da Veiga aparecieron «cerca de mil moedas romanas (medios bronces) dos Sec. III e IV» contenidas en un ánfora fragmentada de la que no aportó numerosas descripciones (Formosinho, 1997: 60).

Posteriormente, en 1938, ingresan en el Museu Regional de Lagos solo «algunas de las mil y pico de monedas romanas encontradas allí, ocasionalmente, dentro de una vasija de barro. Son medios bronces del Bajo Imperio, predominando los de Honorio, Constancio y Arcadio» (Viana et alii, 1953: 120); demostrando así que el tesoro había sido dividido después de su hallazgo. Lamentablemente no hemos conocido aún ni el motivo, ni las cantidades en las que se realizó esta partición, ni tampoco los agentes que intervinieron en tal hecho. Esta información tampoco ha podido ser contrastada con los registros de entrada de piezas conservados en el Museu Municipal de Lagos, ya que para estas fechas la institución no dispone de tales datos. A nuestro juicio, y a pesar de que varios autores hayan afirmado que el tesoro se incorporó de manera íntegra al museo (Santos, 197r: 95), pensamos que la cantidad ingresada debió ser realmente la de 288 piezas; una cifra expuesta en estudios posteriores, y que creemos responde a la expresión «algunas de las mil y pico» que recogen A. Viana, J. Formosinho y O. Ferreira (Viana et alii, 1953: 120).

En 1971, M.L. dos Santos nos proporciona en su capítulo dedicado a Boca do Rio una descripción bastante completa del recipiente donde fueron halladas las monedas (Santos, I971: 89, fig. 2I). La autora también incorporó una imagen de este (Santos, 197r: fig. I2) que nos ha permitido identificar el contenedor con la forma Beltrán 72 (figura 4). Este tipo anfórico había sido tradicionalmente atribuido 


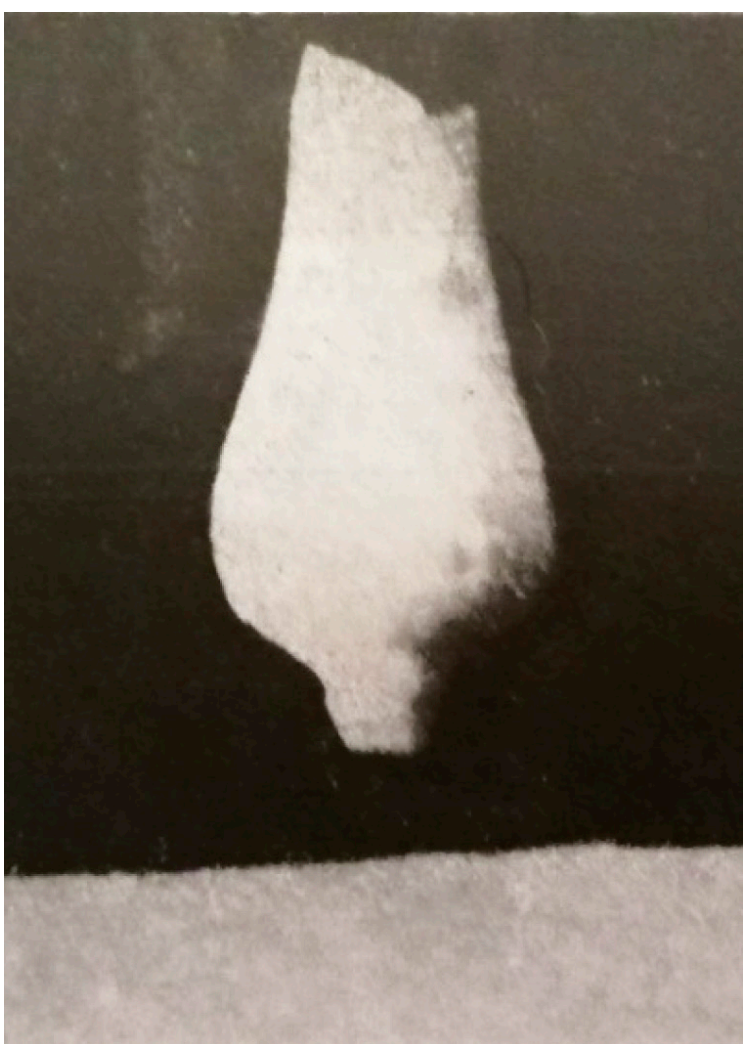

Figura 4. Ánfora fragmentada que contenía el conjunto monetario de Boca do Rio (Santos, 1971: 103, fig. 21)

Figure 4. Fragmented amphora that contained the Boca do Rio hoard (Santos, 1971: 103, fig. 21)

a talleres lusitanos ligados a la producción de preparados piscícolas. Incuso otros autores habían acotado espacialmente la producción de esta forma al Algarve más occidental, en concreto a los territorios del actual Loulé; situado relativamente próximo a Budens y donde se han identificado varios yacimientos dedicados a la producción de conservas de pescado (Diogo, 1987: 184). Sin embargo, recientes estudios y hallazgos han demostrado que el tipo Beltrán 72 es una producción de la Bética (parte oriental y bahía de Cádiz) también destinada al transporte de preparados piscícolas (Bernal-Casasola, 200r: 286; Fabião, 2004: 297). D. Bernal-Casasola estima que este tipo empezó a fabricarse ya en el siglo III, manteniéndose a lo largo del siglo Iv y hasta bien avanzado el siglo v (Bernal-Casasola, 20or: 286). Una cronología que concuerda perfectamente con el consumo de otros productos importados documentados en Boca do Rio (Bernardes y Medeiros, 2016: 280), y la posterior reutilización del ánfora como contenedor de monedas.
En los años 8o del siglo pasado el conjunto monetario es revisado por A. Marques de Faria, quien realiza un estudio preliminar que no llega a publicarse. Sobre los apuntes de este autor trabajará en los años $90 \mathrm{M}$. Sienes, cuya tesis doctoral — publicada en el año 2000- tratará sobre el uso y circulación de las imitaciones de $\mathrm{AE}_{2}$ en la península ibérica. En el catálogo de tesoros estudiados que proporciona la autora, se puede observar de manera sintética, y a modo de tabla, la composición monetaria del conjunto de piezas conservadas en el Museu de Lagos: un total de 288 ejemplares (Sienes, 2000: 48). A excepción del trabajo de Cepeda, quien también hace una descripción, breve, del tesoro (Cepeda, 2000: I80); el conjunto ha permanecido inédito hasta nuestros días, ya que hasta hoy no se había procedido a una catalogación sistemática de sus piezas, ni tampoco a una contextualización histórica del conjunto. Un hecho que permitiría conocer mejor el uso y circulación de la moneda bajoimperial en los territorios algarvíos.

\subsection{Composición}

Las 284 piezas que presentamos corresponden a ejemplares de bronce acuñados en el último tercio del siglo Iv. Este amplio grupo, formaba parte de un conjunto monetario mucho mayor del que desconocemos toda su composición pero en el intuimos la existencia de piezas de cronología anterior. Los datos aportados por el doctor Formosinho ya hacían referencia a que en el contenido de este tesoro también se hallaban medios bronces del siglo III y monedas acuñadas por el propio Constantino (Formosinho, 1997: 60); no obstante ni él ni M.L. Santos proporcionaron ninguna catalogación de las monedas (Santos, I971: 95). Tengamos en cuenta que la mayor parte de los conjuntos monetarios hallados en contextos rurales lusitanos responden a composiciones de gran variedad, donde se incluyen piezas acuñadas con más de cien años de diferencia (casos de interés son los de Frielas —Cardoso, 1995-1997-, Vale do Mouro - Coixâo et alii, 20I5-,El Saucedo - Cabello Briones, 2008: 228-229- o S. Vitória de Ameixial Sienes Hernando, 2000: 46-47-); por lo tanto, no es para nada extraño que el tesoro de Boca do Rio presentara una composición similar (Sienes, 2000: 4I-50; Cepeda, 2000: 176). 
Por otra parte, tenemos que indicar que el número de piezas estudiado por nosotros es inferior al presentado en estudios previos, en concreto a los realizados por A. Marques de Faria y M. Sienes, quienes realizaron su análisis sobre 288 ejemplares. Hoy no se sabe el paradero de estas cuatro piezas, las cuales presentaban mucho interés para nosotros, sobre todo porque entre ellas existía el ejemplar más reciente del conjunto, cuya cronología era utilizada fiablemente como terminus post quem, y sobre el cual reflexionaremos más tarde.

$\mathrm{Si}$ atendemos a la composición de las 284 monedas, a excepción de la pieza 38 , todas las restantes corresponden a $\mathrm{AE}_{2}$ de diversa cronología. El primer grupo engloba los ejemplares con el reverso de Reparatio Reipvb, emitidos todos entre el 378 y 383 por la dinastía valentiniana (I28 ejemplares/45,07\% sobre el total) y durante los años 383 y 388 por el usurpador Magno Máximo (43 piezas/15,04 \% sobre el total). El segundo corresponde a los $\mathrm{AE}_{2}$ con reverso Gloria Romanorum, acuñado por Teodosio y sus hijos (II3 monedas $/ 38,79 \%$ sobre el total) entre el 392-395. La presencia de ambos grupos puede considerarse equilibrada, ya que las diferencias numéricas entre ambos no son significativas. Esto mismo también se ha observado en Hispania (Cepeda, 200o: I76) y en otros tesoros lusitanos hallados en contextos rurales donde el $\mathrm{AE}_{2}$ ha sido la moneda más representada (figura 5).

Comenzando por las emisiones de la dinastía valentiniana, todos los emperadores se encuentran representados con un claro predominio de Graciano, que es el que más ejemplares ha aportado, seguido de Valentiniano II y Teodosio (figura 6). Por lo que respecta a los centros emisores, el mayor aporte monetario del tipo Reparatio Reipub proviene de cecas occidentales, siendo la más representativa Roma, seguida de la de Arelate, Lugdunum y Aquileia. En este sentido este conjunto se encuentra en total consonancia con el aprovisionamiento monetario hispano, ya que desde el 364 hasta el 392, la mayor parte de las monedas que llegaban a Hispania procedían de talleres occidentales (San Vicente, I999: 717). Durante este periodo también se produce el inicio de un paulatino abastecimiento monetario procedente de cecas orientales (Ripollès, 2002: 2I2). Prueba de ello son varios ejemplares acuñados principalmente por Teodosio en las cecas de Thesalonica, Cyzico y Antioquia. Interesante también es el elevado número de piezas que no han aportado marca de ceca debido al desgaste de la moneda o a un golpe flojo en el proceso de acuñación. Por suerte sí hemos podido identificar el emisor, como así puede comprobarse en la tabla $\mathrm{n}^{\circ}$ 2. Bajo esta misma dinastía fue acuñada la pieza no ${ }^{\circ} 8$, la cual corresponde a un $\mathrm{AE}_{3}$ del tipo Virtus Romanorum emitido por Graciano en la ceca de Roma entre los años 378-383. La presencia de esta pieza demostraría la existencia de diferentes valores en el conjunto absoluto y reforzaría nuestra idea sobre la inclusión de piezas antiguas en el total de ejemplares.

Otro elemento interesante de este grupo es la documentación de imitaciones (dos a nombre de Graciano y otras cuatro de emisores no identificados). La presencia de ejemplares fraudulentos de $\mathrm{AE}_{2}$ del tipo Reparatio Reipub es bastante frecuente en el sur peninsular, sobre todo a nombre de Graciano, emisor que más ejemplares imitados ha proporcionado en la mayor parte de los tesoros hallados en este territorio (Sienes, 2000: 134). Para M. Sienes estas acuñaciones fraudulentas fueron continuas durante la usurpación de Magno Máximo, quien hipotéticamente

\begin{tabular}{|c|c|c|c|c|c|c|c|c|}
\hline Periodos & Boca do Rio & Abicada & Freiria & S. Victoria Ameixial & Torrecaños & Garciaz & La Sevillana & EI Saucedo \\
\hline $378-383$ & 116 & - & 26 & 1633 & 520 & 493 & 18 & 33 \\
\hline 383-387 occ. & 43 & 13 & 13 & 45 & 174 & 231 & 13 & 12 \\
\hline 379-387 Or. & 12 & - & 1 & - & 36 & - & 2 & 6 \\
\hline 383-392 Or. & - & - & - & 45 & 18 & 17 & - & - \\
\hline 393-395 & 113 & 10 & 27 & 1514 & 625 & 740 & 23 & 36 \\
\hline Indeterminadas & - & - & - & 4 & 4 & 4 & 4 & 2 \\
\hline Totales & 284 & 23 & 67 & 3186 & 1404 & 1485 & 69 & 89 \\
\hline
\end{tabular}

Figura 5. Comparación de la composición de varios tesoros hallados en villae de Lusitania

Figure 5. Comparison of the composition of hoards found in lusitanian villae 


\begin{tabular}{|c|c|c|c|c|c|c|c|c|c|c|c|c|}
\hline \multicolumn{13}{|c|}{ Reparatio Reipvb (378-383) } \\
\hline Ceca & TR & LVG & ARE & $A Q$ & $\mathbf{R}$ & SIS & TES & $\mathrm{CYZ}$ & ANT & IMI & IND & Total \\
\hline Graciano & - & 6 & 14 & 6 & 18 & 1 & 2 & - & - & 2 & 18 & 67 \\
\hline Valentiniano II & 1 & - & 1 & 2 & 11 & 1 & 2 & - & - & - & 3 & 21 \\
\hline Teodosio & 1 & - & - & 2 & 5 & 2 & 4 & 1 & 1 & - & 6 & 22 \\
\hline Indeterminados & - & 1 & 3 & - & - & - & - & - & - & 4 & 9 & 17 \\
\hline Total & 2 & 7 & 18 & 10 & 34 & 4 & 8 & 1 & 1 & 6 & 36 & 127 \\
\hline \multicolumn{13}{|c|}{ Reparatio Reipvb (383-388) } \\
\hline Ceca & \multicolumn{2}{|c|}{ LVG } & \multicolumn{2}{|c|}{$\mathrm{ARL}$} & \multicolumn{3}{|c|}{ IND } & & & & & Total \\
\hline Mag. Máximo & \multicolumn{2}{|c|}{7} & \multicolumn{2}{|c|}{18} & \multicolumn{3}{|c|}{18} & & & & & 43 \\
\hline \multicolumn{13}{|c|}{ Gloria Romanorum (392-395) } \\
\hline Ceca & HER & CONS & $\mathrm{CYZ}$ & NIC & ANT & ALE & IND & & & & & Total \\
\hline Teodosio & 1 & 11 & 2 & 12 & 14 & - & 10 & & & & & 50 \\
\hline Arcadio & 1 & 2 & 2 & 11 & 6 & 2 & 3 & & & & & 27 \\
\hline Honorio & 1 & 4 & - & 6 & 13 & - & 7 & & & & & 31 \\
\hline Indeterminados & 1 & - & - & 1 & 3 & - & - & & & & & 5 \\
\hline Total & 4 & 17 & 4 & 30 & 36 & 2 & 18 & & & & & 113 \\
\hline \multicolumn{8}{|c|}{ Total de piezas } & & & & & 283 \\
\hline
\end{tabular}

Figura 6. Composición del conjunto monetario atendiendo a emisores y centros de emisión

Figure 6. Composition of the hoard according to emperors and mints

controló estas producciones (Siennes, 2000: 135-136). No obstante, el motivo que explica estas acuñaciones sigue siendo hoy una incógnita, ya que el volumen de moneda acuñada para estos momentos era muy alto como para considerarlas una respuesta ante la falta de aprovisionamiento.

Por lo que respecta al numerario del usurpador Magno Máximo, su presencia en el conjunto es también significativa con un total de 46 ejemplares. La inclusión de la Diocesis Hispaniarum bajo el dominio de este general britano favoreció que tales territorios fueran rápidamente abastecidos con moneda acuñada en cecas situadas en Galia, sobre todo del taller de Arelate (Cepeda, 2000: 165). Este hecho podemos también comprobarlo en las piezas del usurpador catalogadas en el conjunto, donde Arelate es la ceca más representada (con I8 ejemplares), seguida de la de Lugdunum (8 piezas) (figura 6).

En cuanto a las acuñaciones del tipo Gloria Romanorum, observamos un claro protagonismo de Teodosio seguido por Honorio y Arcadio. Las cecas documentadas son todas orientales, siendo la más representada la de Antioquia, acompañada por la de Nicomedia y la de Constantinopla respectivamente (figura 6). La relevancia de estos tres talleres es la habitual, ya que ha sido también documentado en el resto de hallazgos peninsulares y lusitanos (Figuerola, 1999: 375; San Vicente, 1999: 599-600).
J.J. Cepeda ha expuesto que la abundancia de moneda procedente de estos centros responde básicamente al volumen de emisión de cada taller, ya que eran de todos los orientales los que más oficinas operativas tenían (Cepeda, 2000: I65).

Parece ser que la pieza que cerraba este conjunto era un $\mathrm{AE}_{3}$ del tipo Virtus Exerciti acuñado por Arcadio en la ceca de Cyzico entre el 395-402 (Sienes, 2000: 48, Berdardes y Medeiros, 20I6: 283). Según J.J. Cepeda, la presencia de este tipo de ejemplares en tesoros compuestos mayoritariamente por $\mathrm{AE}_{2}$ podría considerarse incluso extraña, ya que los usuarios preferían por tamaño y peso los ejemplares más antiguos; no obstante su existencia permite a los numísmatas acotar de una manera más precisa la fecha de cierre de los conjuntos monetarios (Cepeda, 2000: 164). Además, el descubrimiento de estos $\mathrm{AE}_{3}$ y $\mathrm{AE}_{4}$ acuñados con posterioridad al 395, junto $\mathrm{AE}_{2}$ anteriores, demuestra que los últimos continuaron circulando mucho tiempo después de su acuñación. Numerosos hallazgos aislados prueban el uso de estos $\mathrm{AE}_{2}$ como moneda corriente y su tesauriación en varios yacimientos durante el siglo $\mathrm{V}$ (Ripollés, 2002: 2I4). Una cronología que se prolonga hasta la siguiente centuria si atendemos al hallazgo de estos ejemplares junto a cerámicas datación precisa (Marot, 2000-2000I: I43, Fabião, 2009: 27; Mora Serrano, 20r6: I40). 


\begin{tabular}{lcccccccc}
\hline Ceca & HER & COS & CYZ & NIC & ANT & ALE & IND & Total \\
\hline N $^{\circ}$ Ejemplares & 4 & 17 & 4 & 30 & 36 & 2 & 18 & 113 \\
\hline Media de los pesos & $4,55 \mathrm{~g}$ & $4,72 \mathrm{~g}$ & $4,72 \mathrm{~g}$ & $4,69 \mathrm{~g}$ & $4,65 \mathrm{~g}$ & $4,50 \mathrm{~g}$ & $4,44 \mathrm{~g}$ & $4,63 \mathrm{~g}$ \\
\hline
\end{tabular}

Figura 7. Comparación de la media de pesos por ceca del tipo Gloria Romanorum

Figure 7. Comparison of weights per mint of the Gloria Romanorum type

J.P. Bernardes e I. Medeiros (2016: 283) consideran que el ocultamiento de este conjunto monetario se produjo en las primeras décadas del siglo $\mathrm{v}$, atendiendo a la presencia del $\mathrm{AE}_{3}$ de Honorio y a que el abandono definitivo de la villa se produce a mediados de esa misma centuria. La data propuesta por ambos autores se ve reforzada por el escaso desgaste observado en numerosas piezas del conjunto; una circunstancia que también puede comprobarse en el peso de las monedas, ya que muchos de los ejemplares presentan pesos muy próximos al patrón teórico de $\mathrm{I} / 6 \mathrm{olb}$ que caracteriza a estas emisiones $\left(5,45^{-5}, 38 \mathrm{~g}\right)$. En la media de los pesos $(4,65 \mathrm{~g})$ de las monedas más recientes del conjunto - $-\mathrm{los} \mathrm{AE}_{2}$ del tipo Gloria Romanorum - puede verificarse tal hecho, es decir, se estima que los ejemplares han perdido a causa de la circulación solo un $\mathrm{I}_{4} \%$ del peso total (figura 7), una cifra muy inferior a la observada en otros casos peninsulares donde piezas similares muestran un desgaste muy acusado (Arévalo y Mora, 2018: 67I). Si consideramos la prolongada circulación que experimentaron algunas de estas piezas, pues muchas han sido halladas en Hispania en contextos de los siglos vi y viI (Mora Serrano, 20I7: I40); la presencia de $\mathrm{AE}_{2}$ con escaso desgaste, y con pesos muy próximos al patrón teórico, puede ser considerada un indicador que demostraría que los ejemplares de Boca do Rio circularon muy poco tiempo antes de ser atesorados; por lo que situar la fecha de su ocultamiento en las primeras décadas o primera mitad del siglo $\mathrm{v}$ nos resulta muy convincente.

\section{Tesoros en villae de Lusitania: ¿abandonos accidentados?}

En Lusitania se conocen un gran número de tesoros hallados en villae (Bost, 1992-1993). Una lectura global sobre ellos puede ayudar a solucionar dos de las cuestiones que se plantean los numísmatas a la hora de evaluar estos conjuntos monetarios: el motivo de la ocultación y el de la no recuperación (figura 8). Por desgracia, muchos de los tesoros hoy conocidos fueron descubiertos de manera fortuita, es decir, sin una metodología arqueológica bien definida que nos impide conocer el momento de su ocultación. No obstante, hallazgos más recientes, acompañados de estudios cerámicos precisos, han proporcionado cronologías muy fiables sobre el uso de la moneda romana en contextos tardíos. Por lo que esta situación nos posibilita reflexionar sobre las dos cuestiones anteriores.

Tradicionalmente muchos de estos tesoros han sido relacionados con el abandono de las villae $\mathrm{du}^{-}$ rante el siglo v. Este hecho ha sido achacado de manera habitual a los episodios violentos que pudieron vivirse en Lusitania a principios de esa misma centuria. J. Arce ha expuesto en varias ocasiones que la usurpación de Constantino III llevó a muchos propietarios rurales hispanos, leales a Honorio, a abandonar sus propiedades e incluso a huir a otras provincias por peligro de sus vidas (Arce, 20I2: 25-27). Una situación que posteriormente se agravaría con la llegada de vándalos, suevos y alanos, quienes según las fuentes, realizaron numerosas razias sobre suelo lusitano y bético (Arce, 20Ir: 53-55). Es lógico pensar que en este clima de incertidumbre y de tensión, muchos de estos propietarios marchasen con los elementos de mayor valor, dejando tras sí otros — con la posible intención de recuperarlos en un futuro- que presentaban un volumen y peso costoso de transportar, como eran varios kilos de moneda de bronce.

Varios de los tesoros hallados en las villae de Lusitania podrían responder a este tipo de ocultamiento. Quizás el más ilustrativo sea el de la villa de Vale do Mouro (Coriscada, Mêda, Guarda), compuesto por más de 4000 monedas de bronce de los siglos III y iv. El conjunto - contenido en un saco y con varias piezas de hierro encima- fue escondido en un espacio dedicado a fragua; por lo 


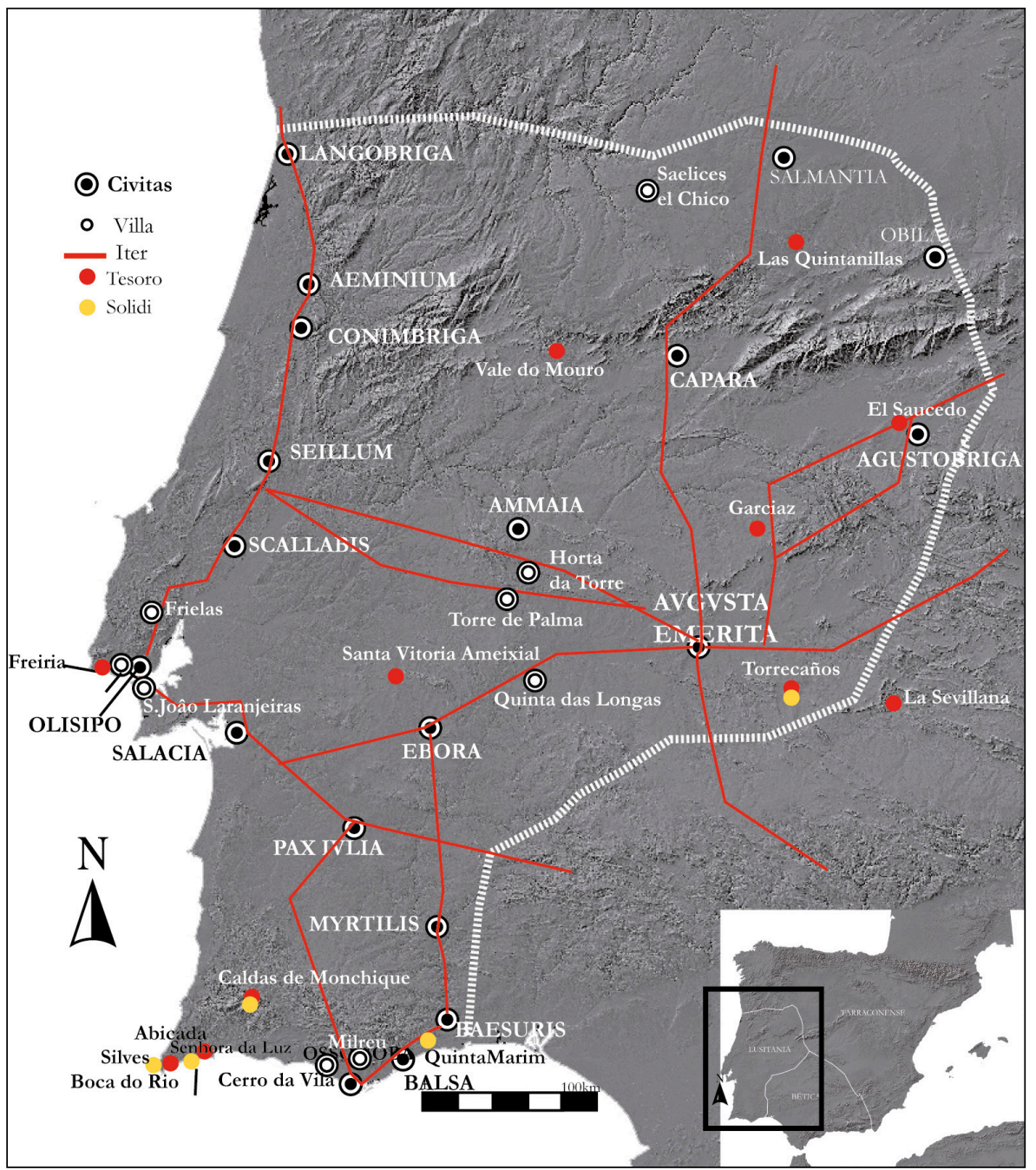

Figura 8. Mapa de Lusitania con las villae mencionadas en el texto, las que han aportado tesoros y los lugares donde han sido halladas monedas de oro

Figure 8. Map of Lusitania with the villae mentioned in the text, the hoards found in villae and the places where gold coins have been found

que suponemos que los usuarios buscaron para el ocultamiento la más absoluta discreción (Coixão et alii, 2015). La villa pierde — sin evidencias cruentassu función residencial y productiva a principios del siglo $\mathrm{v}$, momento que podría coincidir con el ocultamiento del tesoro. A partir de esto, la parte oeste de la villa es reocupada por grupos de carácter humilde que reaprovecharán las estructuras y realizarán algunas labores productivas (Coixão $e t$ alii, 2015: 27). El área donde fue ocultado el conjunto monetario no volvió a sufrir alteraciones, lo que ha permitido la llegada del tesoro a nuestros días. Otros dos tesoros cuyo ocultamiento podría estar relacionado con causas violentas o fortuitas son el de Torrecaños (Guareña, Badajoz) y el de Quinta do
Marím (Olhão, Faro). El primero está compuesto por I $449 \mathrm{AE} 2$, un solidus de Honorio y un anillo de oro (Velázquez, I983); el segundo son unos roo solidi de Honorio hallados en 1786 en un contenedor cerámico (Santos, 1972: 276). Por su contenido, ambos podrían haber sido ocultados a consecuencia de las situaciones violentas anteriormente referidas, sin embargo hoy desconocemos totalmente el contexto arqueológico en el que los dos conjuntos aparecieron.

Autores como A. Chavarría han mostrado que el abandono de las villae durante la primera mitad del siglo $\mathrm{v}$ no puede ser achacado únicamente al factor violento, ya que este proceso debe leerse siempre desde una perspectiva más general con incidencia de otros agentes. Además de la inestabilidad política, 
no se puede olvidar el arruinamiento de los tradicionales propietarios por la crisis de la Annona y la concentración de la propiedad rural por parte de la Iglesia y las nuevas élites (Chavarria, 20I3: I42). Una visión que concuerda con los datos aportados por el registro arqueológico, donde no se ha documentado hasta hoy evidencias, como incendios o destrucciones, que demuestren que el abandono de las villae de Lusitania fue por causas violentas. Este proceso debió ser paulatino y más tarde aprovechado por grupos de carácter humilde, quienes transformaron estas residencias abandonadas en nuevos espacios habitacionales y productivos (Chavarría, 2007: II4).

En esta nueva coyuntura, a la que hay que sumar a principios de siglo $\mathrm{v}$ el fin del aprovisionamiento monetario regular (Cepeda, 2000: 167), tales comunidades se vieron obligadas a generar nuevas estrategias económicas donde primaba la autosuficiencia y subsistencia (Chavarria, 20I4: I43), con la generación incluso de excedentes (Bernardes, 20I4: 134-ז36, Carneiro, 2016: 303). Según J.J. Cepeda, en este proceso de involución hacia economías más simples, la moneda romana de bronce - sobre todo $\mathrm{AE}_{2}$ continuó en uso durante las primeras décadas del siglo v, ya que en Hispania no fue muy efectiva la obligación de su retirada de la circulación como habían promulgado Honorio y Arcadio en el 395 (C.Th. XI, 23, 2 en Cepeda, 2000: I72-I73). No obstante, con el paso del tiempo y en el desarrollo de prácticas económicas sencillas, la moneda quedó relegada a un segundo plano en estas sociedades rurales, siendo utilizada puntualmente las de oro para el pago de rentas o nuevos impuestos, y abandonando totalmente las de bronce para las medianas y pequeñas transacciones, las cuales se solventarían con el uso del truque o los excedentes (Cepeda, 2000: 175).

La circulación de moneda de oro en las villae de Lusitania está atestiguada con un número discreto de hallazgos, lo que probaría la propuesta de J.J. Cepeda. Tengamos en cuenta que estos ejemplares, además de ser fácilmente convertibles, presentaban un uso muy limitado, por lo que su pérdida era bastante complicada. Las villae del sur de la Lusitania han sido los que más piezas de oro han aportado de todas las conocidas en la provincia; por lo que es probable que estas especies monetarias circularan en mayor número por esta área, condicionada sin duda por la costa y sus respectivas actividades productivas. Sin contar el tesoro de Quinta do Marim en Olhão, solidi de Honorio han sido descubiertos en los sitios de Senhora da Luz (Lagos) (Santos, I971: III), Silves (Santos, I972: I02), Caldas de Monchique (Santos, I971: 51) y Cerro da Vila.

Por lo que respecta al bronce, recientes hallazgos demostrarían que la situación era mucho más compleja de la expuesta por J.J. Cepeda. Junto al oro, el bronce romano siguió manteniendo mucha importancia en estas economías rurales, al menos durante el siglo v y principios del vi (Marot, 2000: 150; Depeyrot, 2002: 76, Mora Serrano, 20I6: I40). En las villae de Saelices el Chico (Salamanca) (Dahí y Martín Chamoso, 2005), Horta da Torre (Monforte, Portalegre) (Carneiro, 2016: 295), Quinta das Longas (Elvas, Évora) (Almeida y Carvalho, 2005: 348), São João Laranjeiras (Seixal, Setúbal) (Santos, 2009) o Quinda da Bolacha (Amadora, Lisboa) (Quaresma, 20I7b) han sido documentadas monedas de bronce acuñadas en los siglos III y IV junto a tipos cerámicos comunes e importados datados en los siglos $\mathrm{v}$ y vi. La combinación de ambos materiales prueba dos realidades muy interesantes: en primer lugar que las redes comerciales del momento seguían nutriendo las áreas rurales de Lusitania de varios tipos de mercancía, por lo que al menos hasta finales del siglo $v$ no estaríamos ante una economía volcada únicamente hacia estrategias de subsistencia. En segundo, que las sociedades rurales de esta provincia - al igual que en el resto de Hispania (Mora Serrano, 20I6: I42-I43) — seguían manteniendo las estructuras monetarias conocidas durante el siglo $\mathrm{V}$ e incluso el vi, a pesar de no experimentar una renovación del numerario. Prueba del afán de los usuarios en mantener las estructuras de una economía monetaria son las piezas de bronce partidas halladas en algunos tesoros ocultados en el tránsito del siglo v al vi, como sucede en los llamados A y E de la ciudad de Conimbriga. Estas piezas han sido interpretadas por T. Marot como la respuesta inmediata a la necesidad de divisores con los cuales poder hacer frente a pequeñas transacciones del mundo cotidiano (Marot, 2000-200I: 150).

El uso prolongado de piezas antiguas de bronce en estas sociedades y economías rurales estaría 
regido por un sistema de equivalencias ampliamente conocido, el cual establecería una relación directa de valores entre los productos (mercancías y/o excedentes), las piezas de bronce y la moneda de oro. Un ejemplo de la existencia de estas equivalencias son algunos de los textos conocidos como pizarras visigodas. Estos fueron frecuentes en el noroeste de Lusitania desde el siglo $\mathrm{v}$ hasta el $\mathrm{x}$ y muchos de ellos han sido interpretados como un sistema de contabilidad con fines fiscales, ya que aportan una relación entre usuarios y las cantidades específicas de vino o cereal que los mismos debían contribuir (Díaz Martínez y Martín Viso, 2orr: 234). Bien es cierto que las referencias a la moneda de oro son escasas y según los autores, estas hacen alusión a moneda de cuenta y no física. Sin embargo, esto no impide que tales usuarios no supieran la equivalencia entre el metal amarillo y las cantidades que aportaban en especie. De hecho, quienes han estudiado estos materiales epigráficos han estimado que tal sistema hunde sus raíces en la contabilidad dominical del Bajo Imperio (Cordero Ruiz y Martín Viso, 20I2: 26I), donde el oro era la referencia más fiable para cualquier tipo de cómputo (Carlà, 2009: 275-28I).

Otro ámbito también muy interesante a considerar es la propia legislación. Aquí también pueden observarse equivalencias entre la moneda de bronce y la de oro que pueden darnos información sobre la formación de estos tesoros. En dos leyes del Codex Theodosianus puede comprobarse tales relaciones de valor. En el año 396 se precisaban veinticinco libras de bronce para una sola moneda acuñada en el metal amarillo (C.Th. XI.2I.2); una cantidad que aumenta en las siguientes décadas; ya que en el 424 para una moneda de oro ya eran necesarias cien libras de bronce (C.Th. XI.2I.3). Las cifras irán incrementándose a lo largo de los años, como prueba un edicto de Valentiniano III del 445 (Val. Nov. I6), donde se establecía que un sólido debía ser tasado en 7200 nummi (con un peso teórico de $\mathrm{I}, \mathrm{I} 3 \mathrm{~g}$ ); una estimación que ya se había duplicado en el 493, cuando ya eran necesarios 14400 nummi para una sola moneda de oro (Moordheard, 20I4: 618). Como es evidente, estas equivalencias eran utilizadas de manera frecuente por los collectari, quienes eran los encargados de llevar a cabo las conversiones entre ambos metales. Autores como E. García Vargas han resaltado la importancia de los cambistas en este tipo de operaciones en el Bajo Imperio; un colectivo al que más tarde se sumarán comerciantes de las comunidades orientales residentes en las ciudades occidentales, quienes además de favorecer la circulación de la moneda de oro, también se convertirían en cambistas del bronce (García Vargas, 20II: rog). Partiendo de estos hechos, es más que probable que estos conjuntos monetarios hallados en villae sean depósitos creados por unos usuarios, cuya principal intención era reunir los volúmenes de bronce necesarios para conseguir una o varias monedas de oro. Esto explicaría el abundante volumen de monedas que conforman tales conjuntos, compuestos en su mayoría por ejemplares de pesos altos, lo que permitía a los usuarios conseguir de manera rápida la cantidad necesaria de bronce para adquirir su equivalencia en oro. $A$ priori podría considerarse que tales conjuntos no recuperados son en realidad ejemplos de la incapacidad de los usuarios en reunir las cantidades de bronce marcadas por la legislación y/o los cambistas. Sin embargo, no podemos caer en errores simplistas, pues a pesar de que la totalidad de las piezas que conforman estos conjuntos se hallaban totalmente desmonetizadas por la legislación, estas seguían poseyendo gran estimación entre los usuarios debido su valor intrínseco, de ahí que continuaran en uso en los años posteriores a su desmonetización.

Ya hemos expuesto que tradicionalmente se ha considerado que el ocultamiento de estos conjuntos monetarios ha sido una consecuencia de hechos fortuitos y violentos; sin embargo, aún no existen evidencias claras en el registro arqueológico que demuestren tales circunstancias. De hecho, muchos de estos conjuntos procedentes de villae han sido hallados en estancias abandonadas, mientras que otros ámbitos del mismo lugar seguían activos gracias a los grupos humildes anteriormente mencionados. Esta situación es la que ha sido observada en las villae de Freiria (Cardoso, 1995-1997), de La Sevillana (Aguilar Sáez y Guichard, r993: r9r y ss), de El Saucedo (Cabello Briones, 2008: 228-229) y también de Boca do Rio. El descubrimiento de estos conjuntos en espacios sin actividad nos puede 
hacer pensar que estas monedas fueron escondidas antes de la llegada de tales grupos humildes, probablemente por los anteriores habitantes de las villae; lo que situaría cronológicamente estos ocultamientos en la primera mitad siglo v. Es lógico considerar que si estos grupos humildes hubiesen conocido semejantes cantidades de monedas, las habrían puesto en circulación, ya que como hemos expuesto anteriormente, muchos de ellos continuaron utilizando la moneda de bronce durante casi toda la centuria, incluso en las primeras décadas del siglo vi.

\section{Algunas consideraciones finales}

El tesoro de Boca do Rio es solo un ejemplo más de la enorme cantidad de conjuntos monetarios conservados en fondos de museos a la espera de una revisión y catalogación. Bien es cierto que tanto este caso como muchos otros es resultado de un hallazgo fortuito que no nos ha proporcionado información estratigráfica. No obstante, este hecho no nos ha impedido poder contextualizar el conjunto y ponerlo en relación con otros hallazgos que sí han aportado tales datos.

En los últimos años, la excavación y reinterpretación de muchos yacimientos lusitanos está permitiendo profundizar en las relaciones sociales y económicas desarrolladas tras fin del sistema imperial romano. En este sentido, trabajos de síntesis y reflexión están matizando algunos conceptos generales - como continuidad y ruptura - que tanto han sido utilizados para explicar las transformaciones vividas en Lusitania e Hispania en el periodo post-romano. Los hallazgos demuestran que tales hechos no fueron simples y que su génesis dependió de numerosos factores, algunos de ellos condicionados por el propio territorio. El saber que estas sociedades continuaron usando la moneda romana en un nuevo escenario político y económico nos permite aproximarnos al complejo mundo de las mentalidades, $y$ ser testigos así de un proceso de adaptación y transformación económica que no debió ser nada fácil. Es evidente que aún existen grandes limitaciones ya que nuestro acercamiento a tal escenario es a través del registro arqueológico. Sin embargo, futuros descubrimientos y nuevas metodologías favorecerán lecturas más completas de este periodo histórico.

\section{Catálogo}

Debido al amplio volumen de piezas a describir, hemos optado por configurar la siguiente tabla donde se encuentran de manera sintética todos los datos del conjunto monetario. Las monedas han sido ordenadas por criterio cronológico y por talleres de emisión, comenzando por los situados al oeste y terminado por los de oriente. El primer campo corresponde al número de este catálogo, le siguen el número de inventario del Museu Municipal de Lagos, el tipo monetario, su emisor, la cronología de la emisión, la ceca o taller donde ha sido acuñada la pieza, la marca de emisión del exergo, el tipo monetario, el peso expresado en gramos con dos décimas, el módulo en milímetros, el eje de cuños en horas del reloj y la referencia catalográfica contenida en el volumen IX del RIC. Se ha elaborado varias láminas donde se incluyen una selección de ejemplares. Los números del catálogo marcados con un asterisco corresponden a imitaciones.

\section{Agradecimientos}

Este trabajo de documentación no hubiese sido posible sin la colaboración del personal del Museu Municipal Doutor Jose Formosinho de Lagos, en especial a la arqueóloga responsable, doctora Elena Morán Hernández, y el apoyo de la arqueóloga Maria Helena Dias Simão. Del mismo modo agradecemos los datos proporcionados por el profesor doctor João Pedro Bernardes de la Universidade do Algarve y los comentarios recibidos por los informantes ciegos en el proceso de evaluación del manuscrito. 


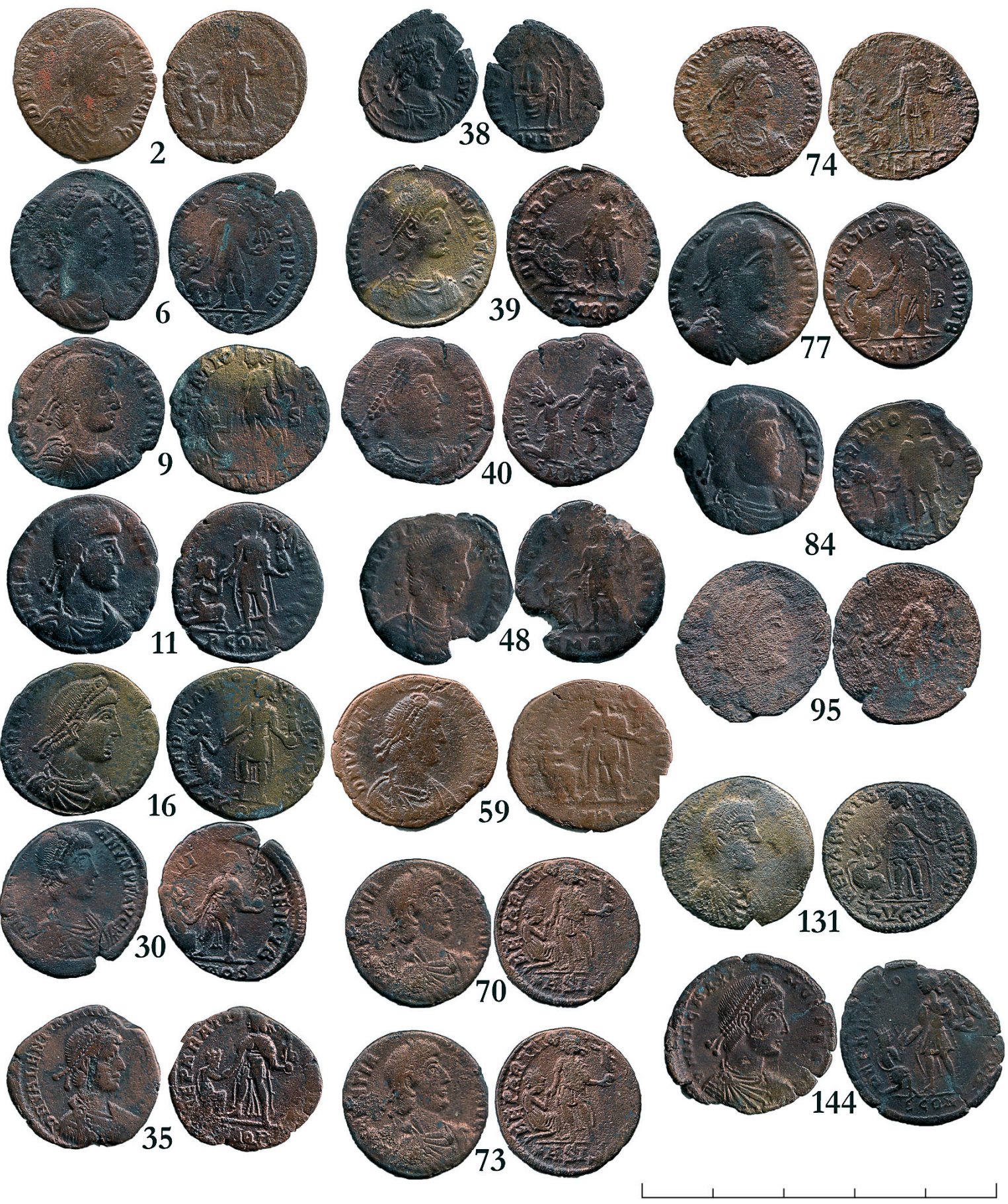

Figura 9. Selección de las piezas más representativas del tesoro

Figure 9. Selection of the most representative pieces of the Boca do Rio hoard 

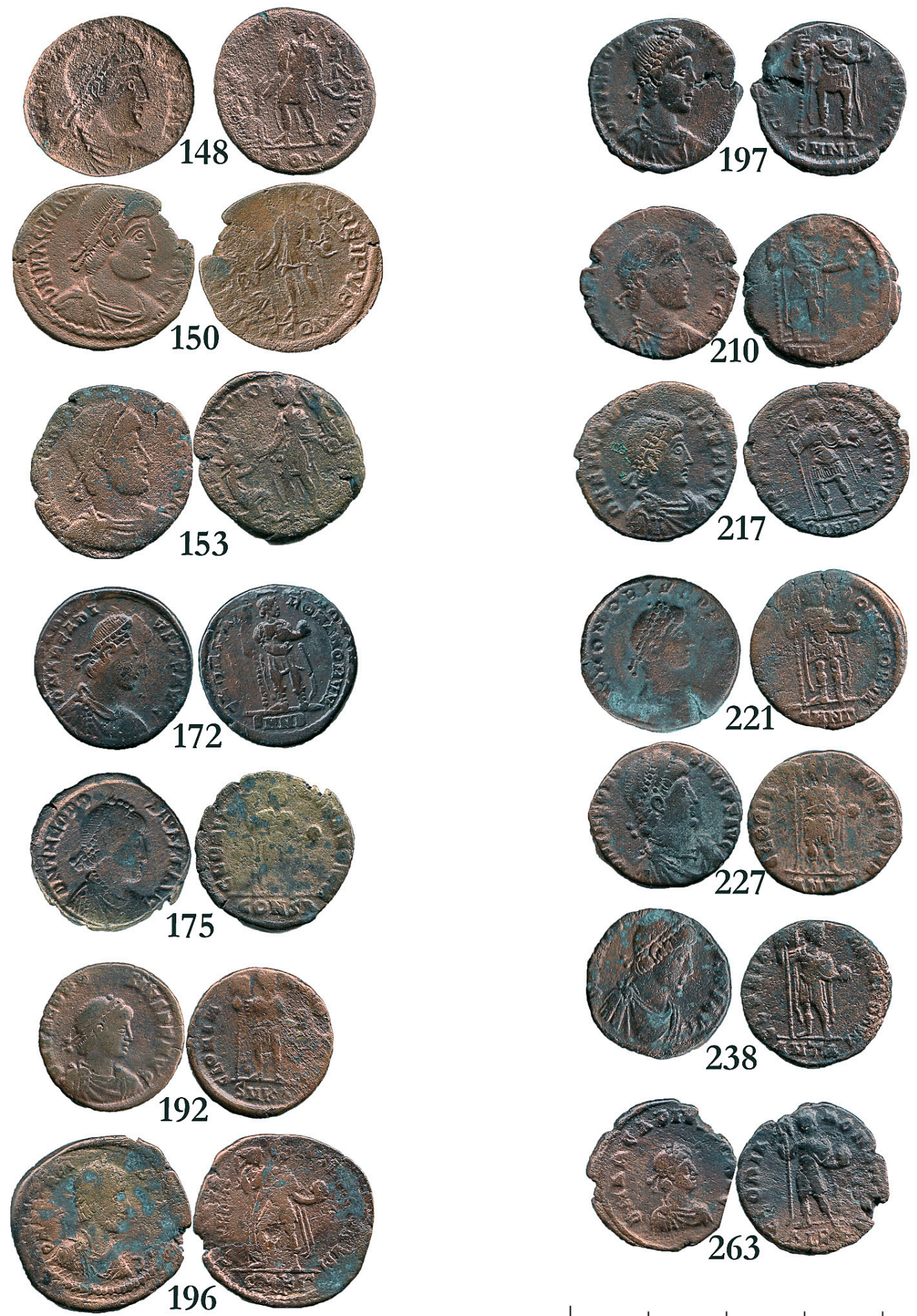

Figura 10. Selección de las piezas más representativas del tesoro

Figure 10. Selection of the most representative pieces of the Boca do Rio hoard 


\begin{tabular}{|c|c|c|c|c|c|c|c|c|c|c|c|}
\hline $\mathbf{N}^{\circ}$ & Inv & Tipo & Emisor & Cron & Ceca & Marca & Tipo Monetario Reverso & $\mathbf{P}$ & $\mathbf{M}$ & $\mathbf{E}$ & RIC IX \\
\hline 1 & 224 & $\mathrm{AE} 2$ & Valent. II & $378-383$ & TR & SMTRP & REPARATIO REIPVB & 3,80 & 21 & 12 & $65 b .1$ \\
\hline 2 & 251 & $\mathrm{AE} 2$ & Teodosio & $378-383$ & TR & SMTRP & REPARATIO REIPVB & 4,89 & 22 & 11 & $65 a .1$ \\
\hline 3 & 150 & AE2 & Graciano & $378-383$ & LVG & LVGS & REPARATIO REIPVB & 5,74 & 22 & 12 & $28 a .4$ \\
\hline 4 & 182 & $\mathrm{AE} 2$ & Graciano & $378-383$ & LVG & LVGP & REPARATIO REIPVB & 4,27 & 22 & 7 & 28a.1 \\
\hline 5 & 186 & $\mathrm{AE} 2$ & Graciano & $378-383$ & LVG & LVGP & REPARATIO REIPVB & 3,85 & 24 & 10 & $28 a .1$ \\
\hline 6 & 105 & AE2 & Graciano & $378-383$ & LVG & LVGS & REPARATIO REIPVB & 3,39 & 23 & 6 & 28 a. 2 \\
\hline 7 & 234 & $\mathrm{AE} 2$ & Graciano & $378-383$ & LVG & LVGP/S & REPARATIO REIPVB & 4,57 & 22 & 12 & 28 a.3 \\
\hline 8 & 250 & $\mathrm{AE} 2$ & Graciano & $378-383$ & LVG & LVGS/S & REPARATIO REIPVB & 7,78 & 24 & 2 & 28 a.3 \\
\hline 9 & 256 & $\mathrm{AE} 2$ & Graciano & $378-383$ & LVG & LVGS/S & REPARATIO REIPVB & 4,78 & 23 & 8 & 28a.4 \\
\hline $10^{*}$ & 111 & $\mathrm{AE2}$ & $\begin{array}{l}\text { Fam. Valen./ } \\
\text { Mag. Max. }\end{array}$ & $378-388$ & LVG & LVGS/S & REPARATIO REIPVB & 5,21 & 23 & 7 & $\begin{array}{c}28 \mathrm{a} .40 \\
32.4\end{array}$ \\
\hline 11 & 4 & $\mathrm{AE} 2$ & Graciano & $378-383$ & ARL & PCON & REPARATIO REIPVB & 4,48 & 23 & 1 & $20 a .1$ \\
\hline 12 & 107 & AE2 & Graciano & $378-383$ & ARL & PCON & REPARATIO REIPVB & 4,70 & 25 & 5 & $20 a .1$ \\
\hline 13 & 145 & AE2 & Graciano & $378-383$ & ARL & PCON & REPARATIO REIPVB & 4,86 & 22 & 10 & $20 a .1$ \\
\hline 14 & 173 & $\mathrm{AE} 2$ & Graciano & $378-383$ & ARL & PCON & REPARATIO REIPVB & 3,94 & 24 & 5 & $20 a .1$ \\
\hline 15 & 43 & AE2 & Graciano & $378-383$ & ARL & SCON & REPARATIO REIPVB & 4,83 & 24 & 7 & $20 a .2$ \\
\hline 16 & 2 & $\mathrm{AE} 2$ & Graciano & $378-383$ & ARL & SCON & REPARATIO REIPVB & 5,30 & 23 & 7 & $20 a .2$ \\
\hline 17 & 50 & $\mathrm{AE} 2$ & Graciano & $378-383$ & $\mathrm{ARL}$ & SCON & REPARATIO REIPVB & 5,61 & 23 & 1 & $20 a .2$ \\
\hline 18 & 66 & $\mathrm{AE} 2$ & Graciano & $378-383$ & ARL & SCON & REPARATIO REIPVB & 4,85 & 22 & 6 & $20 a .2$ \\
\hline 19 & 117 & $\mathrm{AE} 2$ & Graciano & $378-383$ & $\mathrm{ARL}$ & SCON & REPARATIO REIPVB & 4,67 & 23 & 2 & 20a.2 \\
\hline 20 & 162 & $\mathrm{AE} 2$ & Graciano & $378-383$ & ARL & SCON & REPARATIO REIPVB & 3,60 & 25 & 8 & $20 a .2$ \\
\hline 21 & 198 & AE2 & Graciano & $378-383$ & $\mathrm{ARL}$ & SCON & REPARATIO REIPVB & 5,01 & 23 & 7 & $20 a .2$ \\
\hline 22 & 22 & $\mathrm{AE2}$ & Graciano & $378-383$ & ARL & TCON & REPARATIO REIPVB & 4,49 & 22 & 12 & $20 a .3$ \\
\hline 23 & 24 & AE2 & Graciano & $378-383$ & ARL & TCON & REPARATIO REIPVB & 4,28 & 23 & 7 & $20 a .3$ \\
\hline 24 & 61 & AE2 & Graciano & $378-383$ & ARL & TCON & REPARATIO REIPVB & 5,73 & 22 & 6 & $20 a .3$ \\
\hline 25 & 214 & $\mathrm{AE} 2$ & Valent. II & $378-383$ & $\mathrm{ARL}$ & PCON & REPARATIO REIPVB & 4.72 & 25 & 11 & $20 c .1$ \\
\hline 26 & 110 & $\mathrm{AE} 2$ & Fam. Valen. & $378-383$ & $\mathrm{ARL}$ & SCON & REPARATIO REIPVB & 6,10 & 24 & 5 & 20 \\
\hline 27 & 114 & $\mathrm{AE} 2$ & Fam. Valen. & $378-383$ & $\mathrm{ARL}$ & SCON & REPARATIO REIPVB & 4,30 & 23 & 2 & 20 \\
\hline 28 & 116 & $\mathrm{AE} 2$ & Fam. Valen. & $378-383$ & $\mathrm{ARL}$ & $-\mathrm{CON}$ & REPARATIO REIPVB & 3,75 & 23 & 2 & 20 \\
\hline 29 & 6 & $\mathrm{AE} 2$ & Graciano & $378-383$ & $A Q$ & SMAQS & REPARATIO REIPVB & 5,10 & 21 & 1 & $30 a .3$ \\
\hline 30 & 100 & $\mathrm{AE} 2$ & Graciano & $378-383$ & $A Q$ & -MAQS & REPARATIO REIPVB & 4,38 & 21 & 5 & $30 a$ \\
\hline 31 & 112 & AE2 & Graciano & $378-383$ & $A Q$ & SMAQP & REPARATIO REIPVB & 5,32 & 25 & 7 & $30 a .2$ \\
\hline 32 & 118 & $\mathrm{AE} 2$ & Graciano & $378-383$ & $A Q$ & SMAQ-/A & REPARATIO REIPVB & 5,41 & 23 & 7 & $30 a .4$ \\
\hline 33 & 239 & $\mathrm{AE} 2$ & Graciano & $378-383$ & $A Q$ & SMAQS & REPARATIO REIPVB & 5,02 & 22 & 5 & 30a.3 \\
\hline 34 & 113 & $\mathrm{AE} 2$ & Valent. II & $378-383$ & $A Q$ & SMAQP & REPARATIO REIPVB & 3,64 & 24 & 6 & $30 c .2$ \\
\hline 35 & 167 & AE2 & Valent. II & $378-383$ & $A Q$ & SMAQP & REPARATIO REIPVB & 3,40 & 24 & 12 & $30 \mathrm{~b} / \mathrm{c} .2$ \\
\hline 36 & 39 & $\mathrm{AE} 2$ & Teodosio & $378-383$ & $A Q$ & SMAQP & REPARATIO REIPVB & 6,30 & 22 & 2 & $30 d .2$ \\
\hline 37 & 205 & $\mathrm{AE} 2$ & Teodosio & $378-383$ & $A Q$ & SMAQS & REPARATIO REIPVB & 4,92 & 21 & 1 & $30 d .2$ \\
\hline 38 & 174 & $\mathrm{AE} 3$ & Graciano & $378-383$ & ROM & SMRT & VIRTUS ROMARORUM & 1,81 & 18 & 2 & 49.3 \\
\hline 39 & 40 & $\mathrm{AE} 2$ & Graciano & $378-383$ & ROM & SMRP & REPARATIO REIPVB & 6,00 & 24 & 1 & 43a.1 \\
\hline 40 & 168 & $\mathrm{AE} 2$ & Graciano & $378-383$ & ROM & SMRP & REPARATIO REIPVB & 3,91 & 22 & 12 & 43a.1 \\
\hline 41 & 45 & $\mathrm{AE} 2$ & Graciano & $378-383$ & ROM & SMRP? & REPARATIO REIPVB & 5,49 & 22 & 6 & $43 a .1$ \\
\hline 42 & 5 & $\mathrm{AE} 2$ & Graciano & $378-383$ & ROM & SMRB & REPARATIO REIPVB & 6,50 & 21 & 11 & 43а. 2 \\
\hline 43 & 44 & $\mathrm{AE} 2$ & Graciano & $378-383$ & ROM & SMRT & REPARATIO REIPVB & 5,60 & 22 & 6 & 43а.3 \\
\hline 44 & 169 & $\mathrm{AE} 2$ & Graciano & $378-383$ & ROM & SMRT & REPARATIO REIPVB & 6,86 & 22 & 8 & $43 a .3$ \\
\hline 45 & 130 & $\mathrm{AE2}$ & Graciano & $378-383$ & ROM & SMRT & REPARATIO REIPVB & 4,80 & 24 & 6 & 43а.3 \\
\hline 46 & 242 & $\mathrm{AE} 2$ & Graciano & $378-383$ & ROM & SMRT & REPARATIO REIPVB & 5,65 & 22 & 5 & $43 a .3$ \\
\hline 47 & 262 & $\mathrm{AE} 2$ & Graciano & $378-383$ & ROM & SMRT & REPARATIO REIPVB & 5,23 & 23 & 5 & 43a.3 \\
\hline 48 & 35 & $\mathrm{AE} 2$ & Graciano & $378-383$ & ROM & SMRT & REPARATIO REIPVB & 6,27 & 23 & 10 & 43a.3 \\
\hline 49 & 204 & $\mathrm{AE} 2$ & Graciano & $378-383$ & ROM & SMRQ & REPARATIO REIPVB & 6,01 & 23 & 9 & $43 a .4$ \\
\hline 50 & 220 & $\mathrm{AE} 2$ & Graciano & $378-383$ & ROM & SMRQ & REPARATIO REIPVB & 5,82 & 22 & 2 & $43 a .4$ \\
\hline 51 & 231 & $\mathrm{AE} 2$ & Graciano & $378-383$ & ROM & SMRQ & REPARATIO REIPVB & 4,62 & 21 & 11 & $43 a .4$ \\
\hline 52 & 13 & $\mathrm{AE} 2$ & Graciano & $378-383$ & ROM & SMRE & REPARATIO REIPVB & 5,37 & 22 & 8 & $43 a .5$ \\
\hline 53 & 122 & $\mathrm{AE} 2$ & Graciano & $378-383$ & ROM & SMRE & REPARATIO REIPVB & 6,66 & 23 & 2 & $43 a .5$ \\
\hline
\end{tabular}




\begin{tabular}{|c|c|c|c|c|c|c|c|c|c|c|c|}
\hline $\mathbf{N}^{\circ}$ & Inv & Tipo & Emisor & Cron & Ceca & Marca & Tipo Monetario Reverso & $\mathbf{P}$ & $\mathbf{M}$ & $E$ & RIC IX \\
\hline 54 & 199 & $\mathrm{AE} 2$ & Graciano & $378-383$ & ROM & SMRE & REPARATIO REIPVB & 5,70 & 24 & 1 & $43 a .5$ \\
\hline 55 & 245 & $\mathrm{AE} 2$ & Graciano & $378-383$ & ROM & SMRE & REPARATIO REIPVB & 5,02 & 22 & 2 & $43 a .5$ \\
\hline 56 & 268 & $\mathrm{AE} 2$ & Graciano & $378-383$ & ROM & SMR-- & REPARATIO REIPVB & 5,24 & 21 & 12 & $43 a$ \\
\hline 57 & 197 & $\mathrm{AE} 2$ & Valent. II & $378-383$ & ROM & SMRP & REPARATIO REIPVB & 5,63 & 22 & 5 & $43 c .1$ \\
\hline 58 & 143 & $\mathrm{AE} 2$ & Valent. II & $378-383$ & ROM & SMRP & REPARATIO REIPVB & 3,92 & 21 & 10 & $43 c .1$ \\
\hline 59 & 32 & $\mathrm{AE} 2$ & Valent. II & $378-383$ & ROM & SMRB & REPARATIO REIPVB & 5,02 & 25 & 12 & $43 \mathrm{~b} / \mathrm{c}$ \\
\hline 60 & 62 & $\mathrm{AE} 2$ & Valent. II & $378-383$ & ROM & SMRT & REPARATIO REIPVB & 5,96 & 23 & 7 & $43 c .3$ \\
\hline 61 & 166 & $\mathrm{AE} 2$ & Valent. II & $378-383$ & ROM & SMRT & REPARATIO REIPVB & 4,03 & 24 & 7 & 43b.3 \\
\hline 62 & 7 & $\mathrm{AE} 2$ & Valent. II & $378-383$ & ROM & SMRQ & REPARATIO REIPVB & 5,50 & 23 & 1 & $43 \mathrm{~b} / \mathrm{c} .4$ \\
\hline 63 & 1 & $\mathrm{AE} 2$ & Valent. II & $378-383$ & ROM & SMRE & REPARATIO REIPVB & 5,63 & 19 & 10 & $43 b .5$ \\
\hline 64 & 273 & AE2 & Valent. II & $378-383$ & ROM & SMRE & REPARATIO REIPVB & 4,04 & 24 & 11 & $43 c .5$ \\
\hline 65 & 202 & $\mathrm{AE} 2$ & Valent. II & $378-383$ & ROM & SMRE & REPARATIO REIPVB & 4,92 & 22 & 5 & $43 c .5$ \\
\hline 66 & 8 & $\mathrm{AE} 2$ & Valent. II & $378-383$ & ROM & SMRE & REPARATIO REIPVB & 4,63 & 20 & 2 & $43 \mathrm{~b} / \mathrm{c} .5$ \\
\hline 67 & 48 & $\mathrm{AE} 2$ & Valent. II & $378-383$ & ROM & SMRE & REPARATIO REIPVB & 4,55 & 21 & 2 & $43 b / c .5$ \\
\hline 68 & 208 & $\mathrm{AE} 2$ & Teodosio & $378-383$ & ROM & SMRT & REPARATIO REIPVB & 3,92 & 23 & 11 & 43d.3 \\
\hline 69 & 260 & $\mathrm{AE} 2$ & Teodosio & $378-383$ & ROM & SMRE & REPARATIO REIPVB & 4,34 & 23 & 5 & $43 d .5$ \\
\hline 70 & 222 & $\mathrm{AE2}$ & Teodosio & $378-383$ & ROM & SMR- & REPARATIO REIPVB & 4,53 & 21 & 3 & $43 d$ \\
\hline 71 & 190 & AE2 & Teodosio & $378-383$ & ROM & SMR- & REPARATIO REIPVB & 4,70 & 20 & 5 & $43 d$ \\
\hline 72 & 36 & $\mathrm{AE2}$ & Teodosio & $378-383$ & ROM & SMR- & REPARATIO REIPVB & 6,43 & 24 & 5 & 43d \\
\hline 73 & 280 & $\mathrm{AE} 2$ & Graciano & $378-383$ & SIS & *ASISC & REPARATIO REIPVB & 5,25 & 22 & 7 & $27 a .5$ \\
\hline 74 & 38 & $\mathrm{AE} 2$ & Valent. II & $378-383$ & SIS & ASISC & REPARATIO REIPVB & 5,57 & 22 & 8 & $26 c .1$ \\
\hline 75 & 59 & $\mathrm{AE} 2$ & Teodosio & $378-383$ & SIS & ASISC. & REPARATIO REIPVB & 4,13 & 20 & 11 & 26 c. 3 \\
\hline 76 & 189 & $\mathrm{AE} 2$ & Teodosio & $378-383$ & SIS & ASISC. & REPARATIO REIPVB & 4,67 & 23 & 7 & $26 c .3$ \\
\hline 77 & 255 & $\mathrm{AE} 2$ & Graciano & $378-383$ & THE & SMTES/B & REPARATIO REIPVB & 5,22 & 20 & 12 & $37 a .1$ \\
\hline 78 & 124 & $\mathrm{AE2}$ & Valent. II & $378-383$ & THE & SMTHES/A & REPARATIO REIPVB & 4,03 & 24 & 12 & $37 b .1$ \\
\hline 79 & 141 & $\mathrm{AE2}$ & Valent. II & $378-383$ & THE & SMT-/A & REPARATIO REIPVB & 4,90 & 20 & 6 & $37 c .1$ \\
\hline 80 & 269 & $\mathrm{AE} 2$ & Teodosio & $378-383$ & THE & SMTES/A & REPARATIO REIPVB & 5,45 & 22 & 1 & 37d.1 \\
\hline 81 & 271 & $\mathrm{AE} 2$ & Teodosio & $378-383$ & THE & SMTES/A & REPARATIO REIPVB & 7,01 & 22 & 1 & 37d.1 \\
\hline 82 & 236 & $\mathrm{AE} 2$ & Teodosio & $378-383$ & THE & $\mathrm{SMT} / \Delta$ & REPARATIO REIPVB & 4,82 & 23 & 7 & $37 d .4$ \\
\hline 83 & 257 & $A E 2$ & Teodosio & $378-383$ & THE & SMTES/ $\triangle$ & REPARATIO REIPVB & 4,46 & 24 & 10 & $37 d .4$ \\
\hline 84 & 163 & $\mathrm{AE} 2$ & Graciano & $378-383$ & ANT & ANTA & REPARATIO REIPVB & 3,62 & 24 & 2 & $42 a .1$ \\
\hline 85 & 3 & $\mathrm{AE} 2$ & Teodosio & $378-383$ & ANT & ANTI & REPARATIO REIPVB & 6,54 & 21 & 10 & $42 \mathrm{~d} .1$ \\
\hline 86 & 272 & $\mathrm{AE} 2$ & Graciano & $378-383$ & $\mathrm{NV}$ & SM-- & REPARATIO REIPVB & 6,50 & 22 & 1 & -- \\
\hline 87 & 201 & $\mathrm{AE} 2$ & Graciano & $378-383$ & NV & SM-- & REPARATIO REIPVB & 4,12 & 22 & 5 & -- \\
\hline 88 & 115 & $\mathrm{AE} 2$ & Graciano & $378-383$ & NV & SM--- & REPARATIO REIPVB & 4,68 & 23 & 7 & -- \\
\hline 89 & 246 & $\mathrm{AE} 2$ & Graciano & $378-383$ & NV & $---S$ & REPARATIO REIPVB & 3,86 & 25 & 6 & -- \\
\hline 90 & 282 & $\mathrm{AE2}$ & Teodosio & $378-383$ & NV & SM-- & REPARATIO REIPVB & 4,68 & 22 & 5 & -- \\
\hline 91 & 253 & AE2 & Graciano & $378-383$ & NV & & REPARATIO REIPVB & 4,56 & 25 & 6 & -- \\
\hline 92 & 235 & $\mathrm{AE} 2$ & Graciano & $378-383$ & NV & & REPARATIO REIPVB & 4,76 & 22 & 2 & -- \\
\hline 93 & 9 & AE2 & Graciano & $378-383$ & NV & & REPARATIO REIPVB & 4,23 & 24 & 5 & -- \\
\hline 94 & 10 & AE2 & Graciano & $378-383$ & NV & & REPARATIO REIPVB & 6,00 & 21 & 5 & -- \\
\hline 95 & 23 & AE2 & Graciano & $378-383$ & NV & & REPARATIO REIPVB & 5,01 & 22 & 10 & -- \\
\hline 96 & 33 & $\mathrm{AE} 2$ & Graciano & $378-383$ & NV & & REPARATIO REIPVB & 5,05 & 25 & 6 & -- \\
\hline 97 & 73 & $\mathrm{AE} 2$ & Graciano & $378-383$ & NV & & REPARATIO REIPVB & 5,88 & 22 & 5 & -- \\
\hline 98 & 131 & $\mathrm{AE} 2$ & Graciano & $378-383$ & NV & & REPARATIO REIPVB & 4,69 & 23 & 5 & -- \\
\hline 99 & 133 & $\mathrm{AE} 2$ & Graciano & $378-383$ & NV & & REPARATIO REIPVB & 4,10 & 25 & 2 & -- \\
\hline 100 & 142 & $\mathrm{AE} 2$ & Graciano & $378-383$ & NV & & REPARATIO REIPVB & 4,82 & 21 & 2 & -- \\
\hline 101 & 161 & $\mathrm{AE} 2$ & Graciano & $378-383$ & NV & & REPARATIO REIPVB & 5,30 & 22 & 11 & -- \\
\hline 102 & 178 & $\mathrm{AE} 2$ & Graciano & $378-383$ & NV & & REPARATIO REIPVB & 4,40 & 23 & 2 & -- \\
\hline 103 & 192 & $\mathrm{AE} 2$ & Graciano & $378-383$ & NV & & REPARATIO REIPVB & 4,63 & 24 & 2 & -- \\
\hline 104 & 195 & $\mathrm{AE} 2$ & Graciano & $378-383$ & NV & & REPARATIO REIPVB & 3.98 & 23 & 12 & -- \\
\hline 105 & 196 & AE2 & Graciano & $378-383$ & NV & & REPARATIO REIPVB & 4,90 & 24 & 1 & - \\
\hline 106 & 254 & $\mathrm{AE} 2$ & Graciano & $378-383$ & NV & & REPARATIO REIPVB & 3,97 & 23 & 5 & -- \\
\hline 107 & 47 & $\mathrm{AE2}$ & Valent. II & $378-383$ & $\mathrm{NV}$ & & REPARATIO REIPVB & 5,18 & 22 & 2 & -- \\
\hline
\end{tabular}




\begin{tabular}{|c|c|c|c|c|c|c|c|c|c|c|c|}
\hline $\mathbf{N}^{\circ}$ & Inv & Tipo & Emisor & Cron & Ceca & Marca & Tipo Monetario Reverso & $\mathbf{P}$ & $\mathbf{M}$ & $\mathbf{E}$ & RIC IX \\
\hline 108 & 177 & $\mathrm{AE} 2$ & Valent. II & $378-383$ & NV & & REPARATIO REIPVB & 4,30 & 23 & 1 & -- \\
\hline 109 & 223 & $\mathrm{AE} 2$ & Valent. II & $378-383$ & NV & & REPARATIO REIPVB & 4,01 & 21 & 5 & -- \\
\hline 110 & 259 & $\mathrm{AE} 2$ & Teodosio & $378-383$ & NV & & REPARATIO REIPVB & 5,02 & 20 & 2 & -- \\
\hline 111 & 172 & $\mathrm{AE} 2$ & Teodosio & $378-383$ & NV & & REPARATIO REIPVB & 5,12 & 22 & 7 & -- \\
\hline 112 & 247 & $\mathrm{AE} 2$ & Teodosio & $378-383$ & NV & $---S / \Gamma$ & REPARATIO REIPVB & 3,79 & 22 & 5 & -- \\
\hline 113 & 200 & AE2 & Teodosio & $378-383$ & NV & & REPARATIO REIPVB & 4,90 & 22 & 5 & -- \\
\hline 114 & 123 & $\mathrm{AE} 2$ & Teodosio & $378-383$ & NV & & REPARATIO REIPVB & 4,90 & 25 & 11 & -- \\
\hline 115 & 209 & AE2 & Fam. Valen. & $378-383$ & ROM & SMR-- & REPARATIO REIPVB & 4.92 & 20 & 10 & 43 \\
\hline 116 & 46 & $\mathrm{AE} 2$ & Fam. Valen. & $378-383$ & $\mathrm{AQ}$ & SMAQS & REPARATIO REIPVB & 5,73 & 23 & 1 & 30.3 \\
\hline 117 & 49 & $\mathrm{AE} 2$ & Fam. Valen. & $378-383$ & NV & & REPARATIO REIPVB & 5,50 & 21 & 5 & -- \\
\hline 118 & 121 & AE2 & Fam. Valen. & $378-383$ & NV & & REPARATIO REIPVB & 5,90 & 24 & 2 & -- \\
\hline 119 & 135 & AE2 & Fam. Valen. & $378-383$ & NV & & REPARATIO REIPVB & 4,90 & 23 & 6 & -- \\
\hline 120 & 176 & $\mathrm{AE} 2$ & Fam. Valen. & $378-383$ & NV & & REPARATIO REIPVB & 3,70 & 22 & 5 & -- \\
\hline 121 & 215 & AE2 & Fam. Valen. & $378-383$ & NV & & REPARATIO REIPVB & 4,67 & 24 & 6 & -- \\
\hline $122^{*}$ & 248 & $\mathrm{AE} 2$ & Fam. Valen. & $378-383$ & NV & & REPARATIO REIPVB & 4,68 & 20 & 5 & \\
\hline 123 & 284 & $\mathrm{AE} 2$ & Fam. Valen. & $378-383$ & NV & & REPARATIO REIPVB & 5,09 & 23 & 2 & -- \\
\hline 124 & 191 & AE2 & Fam. Valen. & $378-383$ & NV & & REPARATIO REIPVB & 3,28 & 20 & 11 & -- \\
\hline $125^{*}$ & 193 & $\mathrm{AE} 2$ & Fam. Valen. & $378-383$ & NV & & REPARATIO REIPVB & 5,65 & 23 & 2 & -- \\
\hline 126 & 71 & AE2 & Fam. Valen. & $378-383$ & NV & & REPARATIO REIPVB & 5,18 & 22 & 12 & -- \\
\hline 127 & 80 & $\mathrm{AE} 2$ & Fam. Valen. & $378-383$ & NV & & REPARATIO REIPVB & 5,45 & 20 & 2 & -- \\
\hline 128 & 42 & $\mathrm{AE} 2$ & Mag. Max. & $383-388$ & LVG & LVGP & REPARATIO REIPVUB & 5,04 & 24 & 2 & 32.1 \\
\hline 129 & 144 & AE2 & Mag. Max. & $383-388$ & LVG & LVGP & REPARATIO REIPVUB & 4,80 & 22 & 7 & 32.1 \\
\hline 130 & 119 & $\mathrm{AE} 2$ & Mag. Max. & $383-388$ & LVG & LVGS & REPARATIO REIPVUB & 3.66 & 23 & 7 & 32.2 \\
\hline $131^{*}$ & 125 & $\mathrm{AE} 2$ & Mag. Max. & $383-388$ & LVG & LVGS & REPARATIO REIPVUB & 5,27 & 23 & 2 & 32.2 \\
\hline 132 & 126 & AE2 & Mag. Max. & $383-388$ & LVG & LVGS & REPARATIO REIPVUB & 5,44 & 22 & 8 & 32.2 \\
\hline 133 & 249 & $\mathrm{AE} 2$ & Mag. Max. & $383-388$ & LVG & LVGS & REPARATIO REIPVUB & 5,41 & 22 & 11 & 32.2 \\
\hline 134 & 274 & $\mathrm{AE} 2$ & Mag. Max. & $383-388$ & LVG & LVGS & REPARATIO REIPVUB & 5,83 & 24 & 7 & 32.2 \\
\hline 135 & 281 & $\mathrm{AE} 2$ & Mag. Max. & $378-383$ & $A R L$ & PCON & REPARATIO REIPVUB & 3,92 & 23 & 5 & $26 a .1$ \\
\hline 136 & 63 & $\mathrm{AE} 2$ & Mag. Max. & $383-388$ & $A R L$ & PCON & REPARATIO REIPVUB & 6,29 & 21 & 6 & $26 a .1$ \\
\hline $137^{*}$ & 207 & $\mathrm{AE} 2$ & Mag. Max. & $383-388$ & ARL & PCON & REPARATIO REIPVUB & 4,72 & 24 & 10 & $26 a .1$ \\
\hline 138 & 75 & $\mathrm{AE} 2$ & Mag. Max. & $383-388$ & ARL & PCON & REPARATIO REIPVUB & 5,90 & 23 & 12 & 26 a.1 \\
\hline 139 & 34 & AE2 & Mag. Max. & $383-388$ & $\mathrm{ARL}$ & SCON & REPARATIO REIPVUB & 4,75 & 22 & 1 & 26a.2 \\
\hline $140^{*}$ & 89 & $\mathrm{AE} 2$ & Mag. Max. & $388-383$ & ARL & SCON & REPARATIO REIPVUB & 3,99 & 23 & 2 & 26a.2 \\
\hline 141 & 283 & $\mathrm{AE} 2$ & Mag. Max. & $378-383$ & $\mathrm{ARL}$ & SCON & REPARATIO REIPVUB & 4,65 & 23 & 5 & 26a.2 \\
\hline 142 & 96 & AE2 & Mag. Max. & $388-383$ & ARL & $--\mathrm{ON}$ & REPARATIO REIPVUB & 4,46 & 22 & 10 & 26a.2 \\
\hline 143 & 53 & $\mathrm{AE} 2$ & Mag. Max. & $383-388$ & $\mathrm{ARL}$ & SCON & REPARATIO REIPVUB & 6,63 & 24 & 5 & 26a.2 \\
\hline 144 & 127 & $\mathrm{AE} 2$ & Mag. Max. & $383-388$ & $A R L$ & SCON & REPARATIO REIPVUB & 4,67 & 25 & 5 & 26a.2 \\
\hline 145 & 210 & AE2 & Mag. Max. & $383-388$ & $\mathrm{ARL}$ & SCON & REPARATIO REIPVUB & 5,47 & 22 & 6 & 26a.2 \\
\hline 146 & 252 & $\mathrm{AE} 2$ & Mag. Max. & $383-388$ & ARL & SCO--- & REPARATIO REIPVUB & 4,35 & 23 & 7 & 26a.2 \\
\hline 147 & 221 & $\mathrm{AE} 2$ & Mag. Max. & $383-388$ & ARL & SCON & REPARATIO REIPVUB & 3,82 & 23 & 8 & 26a.2 \\
\hline 148 & 241 & $\mathrm{AE} 2$ & Mag. Max. & $383-388$ & ARL & SCON & REPARATIO REIPVUB & 5,15 & 21 & 5 & 26a.2 \\
\hline 149 & 270 & $\mathrm{AE} 2$ & Mag. Max. & $383-388$ & ARL & TCON & REPARATIO REIPVUB & 3,78 & 22 & 2 & 26a.3 \\
\hline 150 & 30 & AE2 & Mag. Max. & $383-388$ & $\mathrm{ARL}$ & TCON & REPARATIO REIPVUB & 5,01 & 24 & 11 & $26 a .3$ \\
\hline $151^{*}$ & 21 & $\mathrm{AE} 2$ & Mag. Max. & $383-388$ & ARL & $-\mathrm{CON}$ & REPARATIO REIPVUB & 5,40 & 24 & 11 & $26 a$ \\
\hline 152 & 37 & $\mathrm{AE} 2$ & Mag. Max. & $378-383$ & ARL & $-\mathrm{CON}$ & REPARATIO REIPVUB & 3,57 & 18 & 10 & -- \\
\hline 153 & 129 & $\mathrm{AE} 2$ & Mag. Max. & $383-388$ & NV & & REPARATIO REIPVUB & 4,90 & 23 & 6 & -- \\
\hline 154 & 120 & $\mathrm{AE} 2$ & Mag. Max. & $383-388$ & NV & & REPARATIO REIPVUB & 5,02 & 22 & 6 & -- \\
\hline 155 & 74 & $\mathrm{AE} 2$ & Mag. Max. & $383-388$ & NV & & REPARATIO REIPVUB & 5,91 & 21 & 10 & -- \\
\hline 156 & 60 & AE2 & Mag. Max. & $383-388$ & NV & & REPARATIO REIPVUB & 4,79 & 20 & 2 & -- \\
\hline 157 & 16 & $\mathrm{AE} 2$ & Mag. Max. & $383-388$ & NV & & REPARATIO REIPVUB & 5,59 & 23 & 11 & -- \\
\hline 158 & 165 & $\mathrm{AE} 2$ & Mag. Max. & $383-388$ & NV & & REPARATIO REIPVUB & 4,52 & 24 & 5 & -- \\
\hline $159^{*}$ & 180 & AE2 & Mag. Max. & $383-388$ & NV & & REPARATIO REIPVUB & 3,70 & 23 & 11 & -- \\
\hline 160 & 181 & $\mathrm{AE} 2$ & Mag. Max. & $383-388$ & NV & & REPARATIO REIPVUB & 3,25 & 22 & 10 & -- \\
\hline 161 & 188 & AE2 & Mag. Max. & $383-388$ & NV & & REPARATIO REIPVUB & 3,48 & 23 & 2 & -- \\
\hline
\end{tabular}




\begin{tabular}{|c|c|c|c|c|c|c|c|c|c|c|c|}
\hline $\mathbf{N}^{\circ}$ & Inv & Tipo & Emisor & Cron & Ceca & Marca & Tipo Monetario Reverso & $\mathbf{P}$ & $M$ & $\mathbf{E}$ & RIC IX \\
\hline 162 & 194 & AE2 & Mag. Max. & $383-388$ & NV & & REPARATIO REIPVUB & 4,80 & 23 & 10 & -- \\
\hline 163 & 206 & $\mathrm{AE} 2$ & Mag. Max. & $383-388$ & NV & & REPARATIO REIPVUB & 4,07 & 21 & 12 & -- \\
\hline $164^{*}$ & 76 & $\mathrm{AE} 2$ & Mag. Max. & $383-388$ & NV & & REPARATIO REIPVUB & 3,35 & 24 & 2 & -- \\
\hline 165 & 77 & $\mathrm{AE} 2$ & Mag. Max. & $383-388$ & NV & & REPARATIO REIPVUB & 65,9 & 25 & 12 & -- \\
\hline 166 & 78 & $\mathrm{AE} 2$ & Mag. Max. & $383-388$ & NV & & REPARATIO REIPVUB & 4,35 & 23 & 12 & -- \\
\hline 167 & 108 & $\mathrm{AE} 2$ & Mag. Max. & $383-388$ & NV & & REPARATIO REIPVUB & 4,46 & 22 & 2 & -- \\
\hline $168^{*}$ & 203 & $\mathrm{AE} 2$ & Mag. Max. & $383-388$ & NV & & REPARATIO REIPVUB & 4,90 & 21 & 2 & -- \\
\hline 169 & 267 & $\mathrm{AE} 2$ & Mag. Max. & $383-388$ & NV & & REPARATIO REIPVUB & 4,25 & 23 & 10 & -- \\
\hline 170 & 258 & $\mathrm{AE} 2$ & Mag. Max. & $383-388$ & NV & & REPARATIO REIPVUB & 3,82 & 21 & 2 & -- \\
\hline 171 & 237 & $\mathrm{AE} 2$ & Teodosio & $392-395$ & HER & $\mathrm{SMH}-/^{*}$ & GLORIA ROMANORUM & 4,02 & 21 & 6 & 27a.3/4 \\
\hline 172 & 146 & $\mathrm{AE} 2$ & Arcadio & $392-395$ & HER & SMHB & GLORIA ROMANORVM & 5,12 & 21 & 2 & $27 b .2$ \\
\hline 173 & 233 & $\mathrm{AE} 2$ & Honorio & 392-395 & HER & $--\mathrm{HB} / *$ & GLORIA ROMANORUM & 5,43 & 22 & 7 & $27 c .3$ \\
\hline 174 & 225 & $\mathrm{AE} 2$ & Fam. Teod. & $392-395$ & HER & $--\mathrm{HB} / *$ & GLORIA ROMANORVM & 3,65 & 18 & 6 & 27 \\
\hline 175 & 67 & $\mathrm{AE} 2$ & Teodosio & $392-395$ & $\mathrm{CON}$ & CONSA & GLORIA ROMANORUM & 4,02 & 23 & 7 & 88a.1 \\
\hline 176 & 104 & $\mathrm{AE} 2$ & Teodosio & $392-395$ & CON & CONSA & GLORIA ROMANORUM & 5,80 & 22 & 8 & 88a.1 \\
\hline 177 & 106 & $\mathrm{AE} 2$ & Teodosio & $392-395$ & CON & CONSA & GLORIA ROMANORUM & 5,80 & 22 & 7 & 88a.1 \\
\hline 178 & 81 & AE2 & Teodosio & $392-395$ & CON & CONSA & GLORIA ROMANORUM & 5,45 & 24 & 6 & $88 a .1$ \\
\hline 179 & 183 & $\mathrm{AE} 2$ & Teodosio & $392-395$ & CON & CONSA & GLORIA ROMANORUM & 3,70 & 21 & 5 & $88 a .1$ \\
\hline 180 & 68 & AE2 & Teodosio & $392-395$ & CON & CONSA & GLORIA ROMANORUM & 4,58 & 22 & 9 & $88 a .1$ \\
\hline 181 & 278 & $\mathrm{AE} 2$ & Teodosio & $392-395$ & CON & CONSA & GLORIA ROMANORUM & 4,24 & 20 & 8 & $88 a .1$ \\
\hline 182 & 51 & AE2 & Teodosio & 392-395 & CON & CONSB & GLORIA ROMANORUM & 5,57 & 20 & 12 & $88 a .2$ \\
\hline 183 & 136 & AE2 & Teodosio & 392-395 & $\mathrm{CON}$ & CONSB & GLORIA ROMANORUM & 5,36 & 22 & 2 & $88 a .2$ \\
\hline 184 & 171 & $\mathrm{AE} 2$ & Teodosio & 392-395 & CON & CONSB & GLORIA ROMANORUM & 4,21 & 22 & 2 & 88a.2 \\
\hline 185 & 238 & $\mathrm{AE} 2$ & Teodosio & 392-395 & $\mathrm{CON}$ & CONST & GLORIA ROMANORUM & 4,82 & 21 & 5 & 88a.3 \\
\hline 186 & 152 & $\mathrm{AE} 2$ & Arcadio & 392-395 & $\mathrm{CON}$ & CONST & GLORIA ROMANORVM & 4,70 & 20 & 1 & $88 \mathrm{~b} .2$ \\
\hline 187 & 219 & $\mathrm{AE} 2$ & Arcadio & 392-395 & $\mathrm{CON}$ & CONS-- & GLORIA ROMANORVM & 4,40 & 22 & 2 & $88 \mathrm{~b}$ \\
\hline 188 & 279 & AE2 & Honorio & $392-395$ & CON & CONSA & GLORIA ROMANORUM & 3,73 & 22 & 2 & $88 \mathrm{c} .1$ \\
\hline 189 & 17 & $\mathrm{AE} 2$ & Honorio & 392-395 & $\mathrm{CON}$ & CONSA & GLORIA ROMANORUM & 4,71 & 21 & 1 & $88 \mathrm{c} .1$ \\
\hline 190 & 261 & AE2 & Honorio & $392-395$ & CON & CONSA & GLORIA ROMANORUM & 5,12 & 21 & 8 & $88 c .1$ \\
\hline 191 & 217 & AE2 & Honorio & $392-395$ & $\mathrm{CON}$ & CONS-- & GLORIA ROMANORUM & 4,72 & 22 & 5 & $88 c$ \\
\hline 192 & 128 & $\mathrm{AE} 2$ & Teodosio & $392-395$ & CYZ & SMKA & GLORIA ROMANORUM & 4,70 & 20 & 1 & $27 a .1$ \\
\hline 193 & 14 & $\mathrm{AE} 2$ & Teodosio & $392-395$ & $\mathrm{CYZ}$ & SMKA & GLORIA ROMANORUM & 4,00 & 21 & 2 & $27 a .1$ \\
\hline 194 & 85 & $\mathrm{AE} 2$ & Teodosio & $392-395$ & $\mathrm{CYZ}$ & SMKГ & GLORIA ROMANORUM & 4,41 & 22 & 5 & $27 a .3$ \\
\hline 195 & 230 & $\mathrm{AE} 2$ & Arcadio & $392-395$ & $\mathrm{CYZ}$ & SMKГ & GLORIA ROMANORVM & 4,90 & 20 & 2 & $27 b .3$ \\
\hline 196 & 52 & $\mathrm{AE} 2$ & Arcadio & $392-395$ & $\mathrm{CYZ}$ & SMKГ & GLORIA ROMANORVM & 5,60 & 24 & 2 & $27 b .3$ \\
\hline 197 & 147 & $\mathrm{AE} 2$ & Teodosio & 392-395 & $\mathrm{NIC}$ & SMNA & GLORIA ROMANORUM & 3,90 & 20 & 1 & 46a.1 \\
\hline 198 & 82 & $\mathrm{AE} 2$ & Teodosio & $392-395$ & $\mathrm{NIC}$ & SMNA & GLORIA ROMANORUM & 4,47 & 21 & 7 & $46 a .1$ \\
\hline 199 & 93 & $\mathrm{AE} 2$ & Teodosio & $392-395$ & $\mathrm{NIC}$ & SMNA & GLORIA ROMANORUM & 4,80 & 22 & 7 & $46 a .1$ \\
\hline 200 & 149 & AE2 & Teodosio & 392-395 & $\mathrm{NIC}$ & SMNA & GLORIA ROMANORUM & 3,77 & 21 & 11 & $46 a .1$ \\
\hline 201 & 153 & AE2 & Teodosio & $392-395$ & $\mathrm{NIC}$ & SMNA & GLORIA ROMANORUM & 4,31 & 24 & 11 & $46 a .1$ \\
\hline 202 & 160 & AE2 & Teodosio & $392-395$ & $\mathrm{NIC}$ & SMNA & GLORIA ROMANORUM & 4,02 & 21 & 4 & $46 a .1$ \\
\hline 203 & 25 & AE2 & Teodosio & $392-395$ & $\mathrm{NIC}$ & SMNA & GLORIA ROMANORUM & 5,15 & 21 & 2 & $46 a .1$ \\
\hline 204 & 20 & AE2 & Teodosio & $392-395$ & $\mathrm{NIC}$ & SMNA & GLORIA ROMANORUM & 5,41 & 21 & 2 & $46 a .1$ \\
\hline 205 & 243 & AE2 & Teodosio & 392-395 & $\mathrm{NIC}$ & SMNB & GLORIA ROMANORUM & 3,84 & 22 & 7 & $46 a .2$ \\
\hline 206 & 212 & $\mathrm{AE} 2$ & Teodosio & 392-395 & $\mathrm{NIC}$ & SMN-- & GLORIA ROMANORUM & 5,32 & 21 & 2 & $46^{a}$ \\
\hline 207 & 154 & $\mathrm{AE2}$ & Teodosio & $392-395$ & $\mathrm{NIC}$ & SMN- & GLORIA ROMANORUM & 4,84 & 20 & 11 & $46^{a}$ \\
\hline 208 & 170 & $\mathrm{AE} 2$ & Arcadio & 392-395 & $\mathrm{NIC}$ & SMNA & GLORIA ROMANORVM & 4,70 & 22 & 7 & $46 c .1$ \\
\hline 209 & 185 & $\mathrm{AE} 2$ & Arcadio & $392-395$ & $\mathrm{NIC}$ & SMNB & GLORIA ROMANORVM & 4,90 & 21 & 2 & $46 \mathrm{~b} .2$ \\
\hline 210 & 91 & $\mathrm{AE} 2$ & Arcadio & $392-395$ & $\mathrm{NIC}$ & SMNB & GLORIA ROMANORVM & 5,12 & 22 & 7 & $46 \mathrm{~b} .2$ \\
\hline 211 & 56 & $\mathrm{AE} 2$ & Arcadio & 392-395 & $\mathrm{NIC}$ & SMNB & GLORIA ROMANORVM & 4,92 & 21 & 7 & $46 \mathrm{~b} .2$ \\
\hline 212 & 94 & $\mathrm{AE} 2$ & Arcadio & $392-395$ & $\mathrm{NIC}$ & SMNB & GLORIA ROMANORVM & 4,67 & 21 & 7 & $46 \mathrm{~b} .2$ \\
\hline 213 & 92 & AE2 & Arcadio & $392-395$ & $\mathrm{NIC}$ & SMNB & GLORIA ROMANORVM & 4,10 & 20 & 1 & $46 \mathrm{~b} .2$ \\
\hline 214 & 151 & $\mathrm{AE} 2$ & Arcadio & 392-395 & $\mathrm{NIC}$ & SMN- & GLORIA ROMANORVM & 4,30 & 20 & 1 & $46 c$ \\
\hline 215 & 275 & $\mathrm{AE2}$ & Arcadio & $392-395$ & $\mathrm{NIC}$ & $-\mathrm{NB}$ & GLORIA ROMANORVM & 5,97 & 22 & 10 & $46 c .2$ \\
\hline
\end{tabular}




\begin{tabular}{|c|c|c|c|c|c|c|c|c|c|c|c|}
\hline $\mathbf{N}^{\circ}$ & Inv & Tipo & Emisor & Cron & Ceca & Marca & Tipo Monetario Reverso & $\mathbf{P}$ & $\mathbf{M}$ & $\mathbf{E}$ & RIC IX \\
\hline 216 & 11 & $\mathrm{AE} 2$ & Arcadio & $392-395$ & $\mathrm{NIC}$ & SMNB & GLORIA ROMANORVM & 5,51 & 22 & 8 & $46 b .2$ \\
\hline 217 & 18 & $\mathrm{AE} 2$ & Arcadio & $392-395$ & $\mathrm{NIC}$ & $\mathrm{SMNB} / *$ & GLORIA ROMANORVM & 4,30 & 22 & 12 & $46 \mathrm{~b} .2$ \\
\hline 218 & 41 & $\mathrm{AE} 2$ & Arcadio & $392-395$ & $\mathrm{NIC}$ & $\mathrm{SMNB} / *$ & GLORIA ROMANORVM & 5,21 & 21 & 5 & $46 b .2$ \\
\hline 219 & 55 & $\mathrm{AE} 2$ & Honorio & $392-395$ & $\mathrm{NIC}$ & $\mathrm{SMNB} / *$ & GLORIA ROMANORUM & 3,66 & 24 & 2 & $46 c .2$ \\
\hline 220 & 26 & $\mathrm{AE} 2$ & Honorio & 392-395 & $\mathrm{NIC}$ & SMNГ & GLORIA ROMANORUM & 4,93 & 22 & 10 & $46 c .1$ \\
\hline 221 & 99 & $\mathrm{AE} 2$ & Honorio & $392-395$ & $\mathrm{NIC}$ & SMNГ & GLORIA ROMANORUM & 4,88 & 21 & 7 & $46 c .1$ \\
\hline 222 & 102 & $\mathrm{AE} 2$ & Honorio & $392-395$ & $\mathrm{NIC}$ & SMNГ & GLORIA ROMANORUM & 5,02 & 22 & 10 & $46 c .1$ \\
\hline 223 & 65 & $\mathrm{AE} 2$ & Honorio & $392-395$ & $\mathrm{NIC}$ & SMN-- & GLORIA ROMANORUM & 5,69 & 22 & 1 & $46 c$ \\
\hline 224 & 156 & $\mathrm{AE} 2$ & Honorio & $392-395$ & $\mathrm{NIC}$ & SMN-- & GLORIA ROMANORUM & 4,85 & 21 & 6 & $46 c$ \\
\hline 225 & 103 & $\mathrm{AE} 2$ & Fam. Teod. & $392-395$ & $\mathrm{NIC}$ & SMNB & GLORIA ROMANORVM & 3,80 & 22 & 7 & 46 \\
\hline 226 & 148 & $\mathrm{AE} 2$ & Teodosio & $392-395$ & ANT & ANTA & GLORIA ROMANORUM & 4,35 & 20 & 5 & 68a.1 \\
\hline 227 & 15 & $\mathrm{AE} 2$ & Teodosio & $392-395$ & ANT & ANTA & GLORIA ROMANORUM & 5,59 & 21 & 12 & 68 a.1 \\
\hline 228 & 72 & $\mathrm{AE} 2$ & Teodosio & $392-395$ & ANT & ANTA & GLORIA ROMANORUM & 5,01 & 23 & 5 & 68a.1 \\
\hline 229 & 109 & $\mathrm{AE} 2$ & Teodosio & $392-395$ & ANT & ANTA & GLORIA ROMANORUM & 3,51 & 24 & 10 & 68 a.1 \\
\hline 230 & 87 & $\mathrm{AE} 2$ & Teodosio & $392-395$ & ANT & ANTA & GLORIA ROMANORUM & 4,76 & 21 & 6 & 68a.1 \\
\hline 231 & 277 & $\mathrm{AE2}$ & Teodosio & $392-395$ & ANT & ANTA & GLORIA ROMANORUM & 3,92 & 20 & 10 & 68a.1 \\
\hline 232 & 140 & $\mathrm{AE} 2$ & Teodosio & $392-395$ & ANT & ANTA & GLORIA ROMANORUM & 4.00 & 22 & 10 & 68a.1 \\
\hline 233 & 158 & $\mathrm{AE} 2$ & Teodosio & $392-395$ & ANT & ANTA & GLORIA ROMANORUM & 3,32 & 23 & 10 & $68 a .1$ \\
\hline 234 & 216 & $\mathrm{AE} 2$ & Teodosio & $392-395$ & ANT & ANTA & GLORIA ROMANORUM & 5,16 & 22 & 11 & 68a.1 \\
\hline 235 & 226 & $\mathrm{AE} 2$ & Teodosio & $392-395$ & ANT & ANTA & GLORIA ROMANORUM & 3,72 & 20 & 5 & 68a.1 \\
\hline 236 & 232 & $\mathrm{AE} 2$ & Teodosio & $392-395$ & ANT & ANTB & GLORIA ROMANORUM & 4,62 & 21 & 2 & 68a.2 \\
\hline 237 & 54 & $\mathrm{AE} 2$ & Teodosio & $392-395$ & ANT & ANTS & GLORIA ROMANORUM & 5,87 & 22 & 3 & 68a.4 \\
\hline 238 & 31 & AE2 & Teodosio & $392-395$ & ANT & ANTS & GLORIA ROMANORUM & 4,87 & 19 & 10 & $68 a .4$ \\
\hline 239 & 70 & $\mathrm{AE} 2$ & Teodosio & $392-395$ & ANT & ANTA & GLORIA ROMANORUM & 4,57 & 22 & 5 & 68a.4 \\
\hline 240 & 164 & $\mathrm{AE} 2$ & Teodosio & $392-395$ & ANT & ANTS & GLORIA ROMANORUM & 4,56 & 19 & 7 & 68a.4 \\
\hline 241 & 175 & $\mathrm{AE} 2$ & Arcadio & $378-383$ & ANT & ANTA & GLORIA ROMANORVM & 4,90 & 20 & 5 & $68 c .1$ \\
\hline 242 & 157 & $\mathrm{AE} 2$ & Arcadio & 392-395 & ANT & ANTB & GLORIA ROMANORVM & 3,48 & 22 & 5 & $68 c .2$ \\
\hline 243 & 228 & $\mathrm{AE} 2$ & Arcadio & $392-395$ & ANT & ANTB & GLORIA ROMANORVM & 5,29 & 22 & 5 & $68 c .2$ \\
\hline 244 & 84 & $\mathrm{AE} 2$ & Arcadio & $392-395$ & ANT & ANTB & GLORIA ROMANORVM & 4,92 & 19 & 5 & $68 c .2$ \\
\hline 245 & 184 & $\mathrm{AE} 2$ & Arcadio & $392-395$ & ANT & ANT- & GLORIA ROMANORVM & 4,23 & 19 & 11 & $68 c$ \\
\hline 246 & 95 & $\mathrm{AE} 2$ & Arcadio & $392-395$ & ANT & ANT-- & GLORIA ROMANORVM & 5,80 & 23 & 7 & $68 c$ \\
\hline 247 & 12 & $\mathrm{AE} 2$ & Honorio & $392-395$ & ANT & ANTA & GLORIA ROMANORUM & 5,11 & 20 & 2 & $68 \mathrm{e} .1$ \\
\hline 248 & 137 & $\mathrm{AE} 2$ & Honorio & $392-395$ & ANT & ANTI & GLORIA ROMANORUM & 3,43 & 20 & 5 & $68 \mathrm{e} .2$ \\
\hline 249 & 138 & $\mathrm{AE} 2$ & Honorio & $392-395$ & ANT & ANTI & GLORIA ROMANORUM & 4,69 & 18 & 11 & $68 \mathrm{e} .2$ \\
\hline 250 & 139 & $\mathrm{AE} 2$ & Honorio & $392-395$ & ANT & ANTI & GLORIA ROMANORUM & 3,80 & 20 & 2 & $68 \mathrm{e} .2$ \\
\hline 251 & 28 & $\mathrm{AE} 2$ & Honorio & $392-395$ & ANT & ANTI & GLORIA ROMANORUM & 4,44 & 21 & 5 & $68 \mathrm{e} .2$ \\
\hline 252 & 187 & $\mathrm{AE} 2$ & Honorio & $392-395$ & ANT & ANTI & GLORIA ROMANORUM & 4,32 & 22 & 5 & $68 \mathrm{e} .2$ \\
\hline 253 & 218 & $\mathrm{AE} 2$ & Honorio & $392-395$ & ANT & ANTI & GLORIA ROMANORUM & 5,02 & 20 & 11 & $68 \mathrm{e} .2$ \\
\hline 254 & 227 & $\mathrm{AE} 2$ & Honorio & $392-395$ & ANT & ANTI & GLORIA ROMANORUM & 4,80 & 22 & 5 & $68 \mathrm{e} .2$ \\
\hline 255 & 79 & $\mathrm{AE} 2$ & Honorio & $392-395$ & ANT & ANTI & GLORIA ROMANORUM & 4,95 & 21 & 5 & $68 \mathrm{e} .2$ \\
\hline 256 & 90 & $\mathrm{AE2}$ & Honorio & $392-395$ & ANT & ANTI & GLORIA ROMANORUM & 4,81 & 20 & 6 & $68 \mathrm{e} .2$ \\
\hline 257 & 57 & AE2 & Honorio & $393-305$ & ANT & ANTI & GLORIA ROMANORUM & 3,54 & 20 & 3 & $68 \mathrm{e} .2$ \\
\hline 258 & 101 & $\mathrm{AE2}$ & Honorio & $392-395$ & ANT & ANTA & GLORIA ROMANORUM & 4,71 & 20 & 7 & $68 \mathrm{e} .3$ \\
\hline 259 & 98 & $\mathrm{AE} 2$ & Honorio & $392-395$ & ANT & ANTA & GLORIA ROMANORUM & 5,20 & 20 & 10 & $68 \mathrm{e} .3$ \\
\hline 260 & 29 & AE2 & Fam. Teod. & $392-395$ & ANT & ANTA & GLORIA ROMANORUM & 6,76 & 20 & 10 & 68 \\
\hline 261 & 266 & $\mathrm{AE} 2$ & Fam. Teod. & $392-395$ & ANT & ANTA & GLORIA ROMANORVM & 3,93 & 21 & 3 & 68 \\
\hline 262 & 86 & $\mathrm{AE} 2$ & Fam. Teod. & $392-395$ & ANT & ANT- & GLORIA ROMANORVM & 5,14 & 19 & 10 & 68 \\
\hline 263 & 155 & $\mathrm{AE} 2$ & Arcadio & $392-395$ & ALE & ALE-- & GLORIA ROMANORVM & 4,52 & 19 & 2 & $21 \mathrm{~b}$ \\
\hline 264 & 211 & $\mathrm{AE} 2$ & Arcadio & $392-395$ & ALE & $--^{*}$ & GLORIA ROMANORVM & 4,49 & 20 & 5 & -- \\
\hline 265 & 97 & $\mathrm{AE} 2$ & Teodosio & $392-395$ & NV & SM--- & GLORIA ROMANORUM & 4,10 & 21 & 2 & -- \\
\hline 266 & 213 & $\mathrm{AE} 2$ & Arcadio & $392-395$ & NV & SM-- & GLORIA ROMANORVM & 3,63 & 20 & 12 & -- \\
\hline 267 & 64 & $\mathrm{AE} 2$ & Teodosio & $392-395$ & NV & & GLORIA ROMANORUM & 3,94 & 24 & $7 c ̧$ & -- \\
\hline 268 & 134 & $\mathrm{AE} 2$ & Teodosio & $392-395$ & NV & & GLORIA ROMANORUM & 3,74 & 20 & 7 & -- \\
\hline 269 & 264 & $\mathrm{AE} 2$ & Teodosio & $392-395$ & NV & & GLORIA ROMANORUM & 4,21 & 22 & 2 & -- \\
\hline
\end{tabular}




\begin{tabular}{|c|c|c|c|c|c|c|c|c|c|c|c|}
\hline $\mathbf{N}^{\circ}$ & Inv & Tipo & Emisor & Cron & Ceca & Marca & Tipo Monetario Reverso & $\mathbf{P}$ & $\mathbf{M}$ & E & RIC IX \\
\hline 270 & 276 & AE2 & Teodosio & $392-395$ & NV & & GLORIA ROMANORUM & 4,39 & 19 & 5 & -- \\
\hline 271 & 240 & $\mathrm{AE} 2$ & Teodosio & 392-395 & NV & & GLORIA ROMANORUM & 3,35 & 20 & 2 & -- \\
\hline 272 & 69 & $\mathrm{AE} 2$ & Teodosio & $392-395$ & NV & & GLORIA ROMANORUM & 5,36 & 20 & 10 & -- \\
\hline 273 & 83 & $\mathrm{AE} 2$ & Teodosio & 392-395 & NV & & GLORIA ROMANORUM & 4,78 & 20 & 6 & -- \\
\hline 274 & 88 & $\mathrm{AE} 2$ & Teodosio & 392-395 & NV & & GLORIA ROMANORUM & 3,54 & 22 & 5 & -- \\
\hline 275 & 27 & $\mathrm{AE} 2$ & Teodosio & 392-395 & NV & & GLORIA ROMANORUM & 4,70 & 21 & 9 & -- \\
\hline 276 & 265 & $\mathrm{AE} 2$ & Arcadio & 392-395 & NV & & GLORIA ROMANORVM & 3.02 & 20 & 8 & -- \\
\hline 277 & 229 & $\mathrm{AE} 2$ & Arcadio & $392-395$ & $\mathrm{NV}$ & & GLORIA ROMANORVM & 5,80 & 22 & 2 & -- \\
\hline 278 & 159 & $\mathrm{AE} 2$ & Honorio & $392-395$ & NV & SM-- & GLORIA ROMANORUM & 3,82 & 20 & 5 & -- \\
\hline 279 & 19 & $\mathrm{AE} 2$ & Honorio & $392-395$ & NV & $-/^{*}$ & GLORIA ROMANORUM & 4,34 & 24 & 11 & -- \\
\hline 280 & 132 & $\mathrm{AE} 2$ & Honorio & $392-395$ & NV & & GLORIA ROMANORUM & 4,96 & 20 & 7 & -- \\
\hline 281 & 244 & $\mathrm{AE} 2$ & Honorio & $392-395$ & NV & & GLORIA ROMANORUM & 3,84 & 22 & 7 & -- \\
\hline 282 & 263 & AE2 & Honorio & $392-395$ & NV & & GLORIA ROMANORUM & 4,52 & 21 & 2 & -- \\
\hline 283 & 58 & $\mathrm{AE} 2$ & Honorio & 393-305 & NV & & GLORIA ROMANORUM & 5,80 & 20 & 6 & -- \\
\hline 284 & 179 & AE2 & Honorio & $392-395$ & NV & & GLORIA ROMANORUM & 5,60 & 22 & 2 & -- \\
\hline
\end{tabular}

\section{Bibliografía}

Aguilar Sáez, A.y Guichard, P. (1993): Villas romaines d'Estremadure, Doña Maria, La Sevillana et leur environnement. Madrid.

Almeida, M.J. y Carvalho, A. (2005): "Villa romana da Quinta das Longas (Elvas, Portugal): A lixeira baixo-imperial". Revista Portuguesa de Arqueología, 8(I): 299-368.

Alves, F. (1997): "Em torno dos projectos da Boca do Rio e do Océan”. Setúbal Arqueológica, II-I2: $225^{-239}$.

Arce, J. (20II): Bárbaros y romanos en Hispania. 400-507 A.D. Madrid.

Arce, J. (20I2): "Campos, tierras y villae en Hispania (siglos IV-VI)". En L. Caballero, P. Mateos y T. Cordero (eds.): Visigodos y Omeyas: El territorio. Anejos a Archivo Español de Arqueología, LXI. Mérida: 2I-30.

Arévalo, A. y Mora, B. (2018): "Las monedas de las cetariae de Tradvcta. Un ejemplo de circulación monetaria en el estrecho de Gibraltar en la Antigüedad Tardía”. En D. Bernal-Casasola y R. Jiménez-Camino (eds.): Las cetariae de Ivlia Tradvcta. Resultados de las excavaciones arqueológicas en la calle San Nicolás de Algeciras (200I-2006). Cádiz: 655-718.
Carvalho, A. y Almeida M.J. (2004): "Vias e circulação de produtos no SW do conventus emeritensis: o exemplo da Quinta das Longas (Elvas, Portugal)". En J.G. Gorges y T. Nogales (coords.): V Mesa redonda internacional sobre Lusitania romana: las comunicaciones: Cáceres, Facultad de Filosofía y letras, 7,8,9 de noviembre de 2002. Mérida: 369-389.

Bernal-Casasola, D. (200I): "La producción de ánforas en la Bética en el siglo iII y durante el bajo imperio romano". En E. García Vargas (ed.): Congreso Internacional "Ex Baetica amphorae»: conservas, aceite y vino de la Bética en el Imperio Romano. Écija: 239-372. Bernárdes, J.P. (2007): "Boca do Rio, I30 anos depois". Xelb, 7: 34I-354.

Bernardes, J.P. (20I4): "Estructuras de produção no mundo rural do sul da Lusitânia durante a antiguidade tardia (séculos v-viI d.C.)". En S. Gomez Martínez, S. Macias y V. Lopes (ed.), O sudeste peninsular entre Roma e o Islão. Mértola: I24-I37.

Bernardes, J.P. y Medeiros, I.E. (20I6): "Boca do Rio (Budens, Vila do Bispo): novos dados de uma villa piscícola romana”. Revista Portuguesa de Arqueología, 19: 265-286.

Cabello Briones, A.M. (2008): Moneda e Historia en tierras de Talavera de la Reina. Los hallazgos monetarios del yacimiento de El Saucedo (Talavera la Nueva, Toledo). Talavera de la Reina. 
Bost, J.-P. (I992-I993): "Villa y circulación monetaria: hipótesis de trabajo”. Studia Historica. Historia Antigua, IO-II: 219-225.

Cardoso, G. (I995-I997): "Um tesouro monetário do Baixo-império na villa de Freiria (Cascais)”. O Arqueólogo Português, 4 (I3-15): 393-4I3.

Cardoso, G. (2016): Estudio arqueológico de la «villa» romana de Freiria. Tesis doctoral inédita. Universidad de Extremadura.

Carlà, F. (2009): L'oro nella tarda antiquità aspetti economici e sociali. Torino.

Carneiro, A. (2016): "O Alto Alentejo durante Antiguidade Tardia”. En J. De Encarnação, M.C. Lopez, y P. Carvalho (eds.): A Lusitânia entre romanos e bárbaros. Coimbra-Magualde: 283-307.

Carneiro, A. (20I7): "O final das villae na Lusitânia romana. O exemplo da Horta da Torre (Fronteira)". Urbs Regia, 2: 56-59.

Chavarría, A. (2007): El final de las villae en Hispania (siglos $I V-V I I$ d. C.). Tournhout.

Chavarria, A. (2013): “¿Castillos en el aire? Paradigmas interpretativos 'de moda' en la arqueología medieval española”. De Mahoma a Carlomagno: los primeros tiempos (siglos VII-LX). XXXIX Semana de Estudios Medievales de Estrella 39. Pamplona: I3I-I66.

Coixão, A., Pereira, P., y Silvino, T. (2015): "La producción vinícola romana en el Nordeste portugués: los ejemplos de Rumasil I (Murça do Douro) y Vale do Mouro (Coriscada)". En M. Contreras y L.V. Elías (eds.): Lagares rupestres: aportaciones para su investigación. Logroño: 23-28.

Cordero Ruiz, T. y Martín Viso, I. (2012): "Sobre los usos y la cronología de las pizarras numerales: Reflexiones a partir del caso del yacimiento de Valdelobos (Montijo, Badajoz)". Archivo Español de Arqueología, 85: 253-266. https://doi. org/Io.3989/aespa.085.012.0I4

Dahí Elena, S. y Martín Chamoso, M.C. (20I2): “Un vaso de terra sigillata hispánica tardía con decoración singular procedente de la Villa romana de Saelices El chico (Salamanca, España)". Archivo Español de Arqueología, 85: 22I-228. https://doi. org/Io.3989/aespa.085.012.012

Depeyrot, G. (2002): Numismatique Antique et médiévale en Occident. Paris.
Díaz Martínez, P. y Martín Viso, I. (20II): “Una contabilidad esquiva: las pizarras numerales visigodas de San Juan (Salvatierra de Tormes, España)”. En P.Díaz Martínez y I. Martín Viso (eds.): Between taxation and rent: fiscal problems from late Antiquity to early Middle Ages. Bari: 22I-250.

Diogo, A.M.D. (1987): "Quadro tipológico das ânforas de fabrico lusitano". O Arqueólogo Português, 4(5): I79-I9I.

Fabião, C. (2004): "Centros oleiros da Lusitania. Balanço dos conhecimentos e perspectivas de investigação”. En L. Lagóstena y D. Bernal-Casasola (eds.): Figlinae Baeticae: talleres alfareros y producciones cerámicas en la Bética romana (ss. II a.C.-VII d.C.). Cádiz: 379-410.

Fabião, C. (2009): "O ocidente da península Ibérica no século vi: sobre o pentanummium de Justiniano I encontrado na unidade de produção de preparados de peixe da Casa do Gobernador da Torre de Belém, Lisboa”. Apontamentos de Arqueologia e Património, 4: 25-50.

Fabião, C. (20r9): "Estácio da Veiga e a Carta Archaeologica do Algarve (I876-I89I): o nascimento da arqueología portuguesa”. En J. Beltrán, C. Fabião y B. Mora (eds.): La Historia de la arqueologia hispano-portuguesa a debate. Historiografía coleccionismo y gestión arqueológica en España y Portugal. Sevilla: 79-Io4.

Figuerola, M. (1999): Cuatro estudios sobre el $A E_{2}$ teodosiano y su circulación en Hispania. Oxford.

Formosinho, J. (I997): “O Dr. José Formosinho e a arqueologia do Algarve”. En F. Barata (ed.): Noventa Seculos. Entre Serra e o Mar. Lisboa: 59-67.

García-Entero, V. (2006): Los balnea domésticos -ámbito rural y urbano- en la Hispania romana. Madrid.

García Vargas, E. (20II): "Oriental trade in the Iberian peninsula during Late Antiquity $\left(4^{\text {th }}-7^{\text {th }}\right.$ centuries AD): An Archaeological Perspective". En D. Hernández de la Fuente (ed.): Nerw Perspectives on Late Antiquity. Cambridge: 76-II7.

Gorges J.-G. (2008): "L'architecture des villae romaines tardives: la création et le développement du modèle tétrarchique”. En C. Fernández Ochoa, V. García-Entero y F. Gil Sendino (eds.): Las villae tardorromanas en el occidente del Imperio: Arquitectura y función. Gijón: 27-48. 
Lagóstena L. (200I): La producción de salsas y conservas de pescado en Hispania romana. Barcelona.

Maloney, S. y Hale, J. (1996): "The villa of Torre de Palma (Alto Alentejo)”. Journal of Roman Archaeology, 9: 275-294. https://doi.org/ıo.IoI7/ Sio47759400016639

Marot, T. (2000-200I): "La Península Ibérica en los siglos V-VI: consideraciones sobre provisión, circulación y usos monetarios". Pyrenae, 31-32: I33-I6o.

Medeiros, I.E. (20I4-2015): "Conservas de peixe na Lusitânia. O quadro productivo da Boca do Rio e das restantes cetariae do Algarve". Anales de Arqueología Cordobesa, 25-26: II5-I44. https://doi. org/I0.2I07I/acc.voi25-26.64I4

Mora Serrano, B. (2016): "Old and new coins in southern Hispania in the $6^{\text {th }}$ century AD". En J. Chameroy y P.M. Guihard (eds.): Produktion und recyceln von Münzen in der Spätantie. Tagungen: I39-153.

Moorhead, S. (2014): "The coinage of the later Roman Empire". En W.E. Metcalf (ed.): The Oxford Handbook of Greek and Roman Coinage. Oxford: 6oI-632. https://doi.org/Io.Io93/oxfordhb/978or95305746.or3.0033

Quaresma, J.C. (20I7a): "A villa de Frielas na Antiguidade Tardia: evolução estratigráfica entre $c$. 4Io e 525-550 d.C." Medieval Sophia, I9: 431-454.

Quaresma, J.C. (20I7b): "Quinta da Bolacha (Amadora, Lisbonne): La céramique de la villa (dernier tiers du $\mathrm{III}^{\mathrm{e}}$ siècle au premier quart du vi $\mathrm{I}^{\mathrm{e}}$ siècle”. En D. Dixneuf (ed.): LRSCW 5-I. Late Roman coarse wares, cooking wares and amphorae in the Mediterranean. Archaeology and Archaeometry. Alexandria: 43-89.

Ripollès, P.P. (2002): "La moneda romana imperial y su circulación en Hispania”. Archivo Español de Arqueologia, 75: 195-214. http://doi.org/10.3989/ aespa.2002.v75.135
RIC IX: Pearce, J.W.E. (1933): The Roman Imperial Coinage, from Valentininian I to Theodosius I. London. Santos, M.L.E.V.A. (1971): Arqueologia romana do Algarve. I. Lisboa.

Santos, M.L.E.V.A. (1972): Arqueologia romana do Algarve II. Lisboa.

Santos, C.R. (2009): Villa romana da Quinta de São João/Laranjeira: enquadramento estratigráfico dos materiais datantes. Trabalho de Maestrado Inédito. Universidade de Lisboa.

San Vicente, I. (1999): Circulación monetaria en Hispania durante el siglo IV d. C. Madrid.

Sienes Hernando, M. (2000): As imitaçôes de moedas de bronze do século IV d. C na Península Ibérica. Lisboa.

Teichner, F. (2006): “De lo romano a lo árabe. La transición del sur de la provincia de Lusitania a al-Gharb al-Andalus". En A. Chavarria, J. Arce, y J.P. Brogiorlo (eds.): Villas tardoantiguas en el Mediterráneo Occidental. Anejos de Archivo Español de Arqueología, XXXIX. Madrid: 207-220.

Teichner, F. (20I7): "Cerro da Vila: A rural commercial harbour beyon the Pillars of Hercules". En J.M. Campos Carrasco y J. Bermejo Meléndez (eds.) Los Puertos Romanos Atlánticos Béticos y Lusitanos y su relación comercial con el Mediterráneo. Roma: 403-433

Veiga, S.P.M.E. Da (I9ro): "Antiguidades monumentaes do Algarve. Cap. V.Tempos Históricos". O Archeologo Português, I (5): 212-218.

Velázquez, A. (1983): "El tesorillo de Torrecaños de Guarea (Badajoz). Contribución al estudio de la circulación monetaria durante el Bajo Imperio en el territorium emeritense". Augusta Emerita, I. Madrid: 85-190.

Viana, A., Formosinho, J. y Ferreira, O. da V. (1953): "De lo prerromano a lo árabe en el Museo Regional de Lagos". Archivo Español de Arqueología, 26: II $3-\mathrm{I} 38$. 